\section{MedienPädagogik}

www. medienpaed.com
Zeitschrift für

Theorie und Praxis

der Medienbildung

ISSN 1424-3636

Themenheft Nr. 19: Mobile Learning in Widening Contexts: Concepts and Cases

\title{
Eckpunkte einer Didaktik des mobilen Lernens Operationalisierung im Rahmen eines Schulversuchs
}

Ben Bachmair, Maren Risch, Katja Friedrich und Katja Mayer

\section{Überblick über den Beitrag}

Der mobile, miniaturisierte Kommunikations- und Mediencomputer Handy ist eine im Alltag der Kinder und Jugendlichen selbstverständliche Kulturressource. Diese Kulturressource auch bewusst für Lernen in der Schule zu nutzen, war das Ziel des Schulversuchs MyMobile - Handy im Unterricht ${ }^{1}$. Die gemeinnützigen Medienpädagogik-Einrichtung Medien+Bildung.com der Landeszentrale für Medien und Kommunikation (LMK), Rheinland-Pfalz, realisierte den Schulversuch 2010 in sechs Schulen in jeweils einer Klasse. Die jeweilige Fachlehrerin bzw. der Fachlehrer führte den Unterricht zusammen mit einer Medienpädagogin bzw. einem Medienpädagogen von Medien+Bildung.com durch. Der Handyeinsatz fand im Rahmen des lehrer/innengeleiteten Unterrichts des vorgegeben Lehrplan des Landes Rheinland-Pfalz statt. Der lehrer/innengeleitete Unterricht öffnete sich für Episoden situierten Lernens mit dem Handy. Dabei kamen sowohl Handys der Schüler und Schüler/innen als auch Handys zum Einsatz, die die Schüler/innen und Schüler zur Verfügung gestellt bekamen.

Der Beitrag stellt einleitend den theoretisch systematischen Rahmen für eine eigenständige Didaktik des mobilen Lernens vor. Ausgangspunkt ist das Handy als Produkt eines Mobilitätskomplexes, der im Alltag neue Kulturressourcen hat entstehen lassen. Der Gedanke der Kulturressource legt es nahe, das alltägliche Handy auch in der Schule curricular ernst zu nehmen und in vom Lehrplan legitimierte Lernprozesse einzubinden. Verantwortlich für die unterrichtspraktische Umsetzung sind Eckpunkte des mobilen Lernens. Diese Eckpunkt operationalisieren den theoretischen Ansatz, den die London Mobile Learning Group ${ }^{2}$ als kulturökologischen Zugang zum mobilen Lernen vorgestellt hat (Pachler, Bachmair, Cook 2010). Anhand eines Beispiels aus der Grundschule zum Erstlesen und Erstschreiben und einer Mathematik-Unterrichtseinheit einer sechsten Klasse des Gymnasiums stellt der zweite Teil des Beitrags die sechs Eckpunkt einer Didaktik des mobilen Lernens vor. Der dritte Teil erörtert die technischen Nutzungsfunktionen des Handys mit Hilfe von sechs Eckpunkten zur multimedialen Funktionsvielfalt des Handys.

1 http://www.medienundbildung.com/index.php?id=464

2 http://www.londonmobilelearning.net 


\section{Mobiles Lernen ist mehr als nur eine didaktisch intelligente Handy-Nutzung}

Wie immer in der Geschichte der Mediendidaktik entwickelt sich ausserhalb der Schule ein gesellschaftlich technologischer Komplex, in dem neue, d. h. für diesen Komplex spezifische Medien und deren Nutzungsformen entstehen. Das begann mit dem Lichtbild, dessen Einzug in die Schule schon in den 1920er-Jahren eine eigene Zeitschrift diskutierte. 1920 schrieb Gymnasiallehrer Dr. H. Mertel aus München im BILD-ARCHIV (1920, Nr. 1, S. 2-3):

Während, sich «das Wort des Lehrers» «wieder störend als sprechender Zeigestab» «zwischen Auge und Verstand» «drängt», hatte «unterdessen»... die Fotographie und in ihrem Gefolge das Lichtbild seinen Siegeslauf angetreten; aber merkwürdig, die Schule stand gleichgiltig bei Seite. ... alle Welt hielt Vorträge mit Lichtbildern, die Wissenschaft bediente sich der Lichtbildkunst so gut wie die Reklame, nur die Schule, zumal die höhere hielt diesem Neuen ihre Tore verschlossen. ... Genau so liegt die Sache beim Film: Gegenüber der Kinoseuche hat die Schule bislang nur Anklage und Verbote, das einzig wirksame Serum gegen diese Geistespest hat sie nicht in Anwendung gebracht, eben den Film. Doch auch hier scheint jetzt der Bann gebrochen.

Wie sich im ersten Satz des Zitates von Mertel lesen lässt, sah der Gymnasiallehrer eine wichtige didaktische Funktion für das Lichtbild, nämlich die eigene Anschauung eines Sachverhaltes zu ermöglichen. Statt nur der Lehrer/innenrede zu folgen, wären Schüler und Schüler/innen mit Hilfe des Lichtbildes in der Lage, sich per «Auge und Verstand» auf den Lerngegenstand zu konzentrieren. Ersetzt man im obigen Zitat «Kinoseuche» mit Handy-Seuche, dann könnte man/frau sich in Diskussionen in heutigen Lehrer/innenzimmern und auf Elternabenden erfolgreich einmischen.

Was lässt sich aus diesem nicht ganz zufälligen Beispiel als Muster destillieren? Das historische Beispiel ist deshalb nicht zufällig, weil es 1920 im Einleitungsheft einer neuen didaktischen Zeitschrift zu lesen war und damit zur Institutionalisierung der didaktischen Beschäftigung mit einem neuen, gesellschaftlich wichtigen Medium beitrug. Charakteristisch für dieses Muster ist zum einen der zwar vage, aber vorhandene Verweis auf einen gesellschaftlichen Komplex neuer Medien und zum anderen der schulinnovatorische Versuch, diese neuen Medien auch didaktisch zu nutzen. 1920 gehörten das Lichtbild in der Wissenschaft und die "Kinoseuche» zum damaligen gesellschaftlichen Medien-Komplex. Beim Hinweis auf das Lichtbild in der Wissenschaft handelt es sich, modern formuliert, um eine erfolgreiche Ressource im Wissenschaftsprozess, die sich auch als neues Hilfsmittel, also als didaktische Ressource, im Anschauungsunterricht einsetzen lässt. Hinter dem sim- 
plen pädagogischen Bild von der «Kinosucht» verbirgt sich eine Ressource des Alltagslebens; Kino und Film als neue Ressource der Erzählung und der Unterhaltung. Das ist eine Ressource, mit der damalige Didaktiker/innen wenig anzufangen wussten. Verlängert man den Gedanken des gesellschaftlichen Medien- und Technologie-Komplexes und seiner Ressourcen an das Ende des 20. Jahrhunderts trifft man auf den PC- und Internet-Boom mit seinen technologischen wie ökonomischen Erscheinung, in den sich Didaktik und auch die Schulverwaltung erfolgreich eingebracht hat. Nicht nur die Wirtschaft, sondern auch Politik, Bildungsverwaltung und andere gesellschaftliche Gruppen sahen in PC und Internet Ressourcen der Modernisierung und in deren Nutzung eine Kulturtechnik, zu deren Erwerb die Schule beizutragen hatte. Die Bertelmann-Stiftung (1999) agierte hier z. B. als Innovationsagentur, die im Memorandum «Zukunft gewinnen - Bildung erneuern» von 1999 für eine neue Lernkultur eintrat. Das Memorandum nennt als eine von vier Leitlinien eines «Paradigmenwechsels in der Bildung» (S. 21): "Lernwelten erschliessen: neue Medien einsetzen» (S. 25 ff.).

Der Gedanke, Medieninnovationen der Gesellschaft als Kulturressource in der Schule zu verwenden, ist für Pädagogik wie für die Institution der Schule ein Thema, das mit der Vorstellung von Entfremdung des Lernens, der Entfremdung von der direkten Erfahrung und der Entfremdung vom kulturell tradierten Leitmedium des Buches verbunden ist. Janette Böhme (2006) beschreibt diese Entfremdungsvorstellungen mit dem Bild der «Schule als medienresistenter Polis» (S. 39), die am «bildungskulturellen Leitmedium» (S. 17) des Buches festhält. Die «medienresistente» Schule kann sich der Zustimmung der, wie es Jürgen Habermas (1990, S. 14ff.) nannte, «kulturräsonierenden Öffentlichkeit» sicher sein (vgl. Bachmair 2009, S. 19 ff.). Oder doch nicht? Die enorme Geschwindigkeit, mit der die Computer gebundene Informationstechnologie sich in der Schule im Sinne der obigen Leitlinie «Zukunft gewinnen - Bildung erneuern» durchsetzte, scheint dagegen zu sprechen. Wahrscheinlich hat sich die «medienresistente» Schule auf die Aufgabe der gesellschaftliche Innovation eingelassen und gesellschaftlich innovative Medien in der Sphäre der Schule akzeptiert. Für gesellschaftliche Innovation stand bis vor kurzem der Computer und steht heute die Medienkonvergenz mit dem Internet. Da bleiben Pädagogik und Schule nur noch die Abwehr von Unterhaltungsmedien und Unterhaltungsinhalten. Im Moment repräsentiert das Handy diese Unterhaltungsmedien und Unterhaltungsinhalte.

Hat die Schule mit ihrem Handy-Verbot nicht recht, um dem sachbezogenen Lernen wenigstens einen geschützten Raum zu belassen? Es zeichnet sich jedoch ab, dass das Handy unter dem Gesichtspunkt der gesellschaftliche Innovation auch für die Schule hoffähig wird. Stichwort ist hier die Medienkonvergenz mit dem Handy als ständig verfügbarer Schnittstelle zum Internet. Das Handy als sichtbares Instrument eines globalen Mobilitätskomplex und dessen Innovationspotential lässt es deshalb geraten erscheinen, pädagogisch nachzudenken, wie sich denn 
das Handy als Kulturressource didaktisch nutzen lässt. Die im folgenden Beitrag vorgestellten Eckpunkte des mobilen Lernens sind als konkreter Beitrag zu solch einer pädagogischen Reflexion gedacht.

\subsection{Der Mobilitätskomplex in der Perspektive der Schule}

Der Internet-Komplex von vor 10 Jahren wandelte sich im Prozess der Individualisierung zu einem Mobilitätskomplex, bei denen Handy, Smartphones, Tablett-PCs oder sonstige mobile Technologie den ubiquitären, das heisst, den allgegenwärtigen und immerwährenden Zugang und Verfügbarkeit nicht nur zum Internet, sondern zum Medien- und Informationssystem gewährleistet. Die statische Beziehung PC / Internet / Nutzerln wandelt sich zu einer mobilen Beziehung. Mobile Beziehung heisst vor allem, eine individuelle Verfügbarkeit im konvergenten Medienund Informationssystem des Internets mit von Nutzern/-innen generierten Kontexten (Dourish 2004) und Inhalten.

Wie sieht der Mobilitätskomplex in der Perspektive der Schule und der Schüler aus? Hierzu nur einige Stichpunkte. Die breite Diskussion findet sich bei Pachler, Bachmair, Cook (2010, S. 3 ff.). Der Mobilitätskomplex ist alles andere als nur eine technologische Angelegenheit. Technologie ist eingebunden in sozialkulturelle Veränderungen wie die Ausdifferenzierung der Bevölkerung nach Lebensstilen und sozialkulturellen Milieus. In unterschiedlichen Milieus aufzuwachsen führt zu unterschiedlichen Persönlichkeitsmustern und damit auch zu anderen Formen des Lernens und der Lebensgestaltung. In diesem Prozess der Ausdifferenzierung verliert die Schule ihre Definitionsmacht für das, was als Lernen angesehen und geschätzt wird. Informelles Lernen bekommt zunehmend mehr Bedeutung. Das Stichwort Wissensgesellschaft liefert die Brücke zwischen den neuen, im Moment noch informellen Lernformen und dem Mobilitätskomplex. Dazu gehört u.a. die Inszenierung des Banalen, wie Lothar Mikos (2004) eine der Strukturen der Wissensgesellschaft nennt. Dazu gehören auch neue und schulferne Formen des Lernens (Jörissen 2007, Tervooren 2007).

Wesentlich für den Mobilitätskomplex ist die Medienkonvergenz. Das ist das vernetzte und globale Medienangebot (siehe Kleinsteuber, Thomass 2004), mit vernetztem Zugang zu den Medien und einer Mediennutzung in Netzen (siehe Hasebrink u. a. 2004). Wenn man sich informelles, dennoch schulnahes Lernen in der Medienkonvergenz veranschaulichen will, dann genügt ein Blick in Hausaufgabenforen auf YouTube. Das Handy-Video, zum Beispiel, mit der Veranschaulichung des Pythagoras und mit den kommunikativen Folgeketten ist amüsant und zudem eindruckvoll, ob der Ernsthaftigkeit der Beschäftigung mit Mathematik. ${ }^{3}$

3 http://www.youtube.com/watch?v=hbhh-9edn3c\&feature=related 7. Dezember 2006 - Pythagoras' Theorem water-proved (made in Switzerland); aufgenommen am 16. Mai 10 
Wie reagiert die Schule im Moment auf diesen Mobilitätskomplex, bei dem das Handy die Spitze des Eisberges ist? Im Moment steht bei den Schulen im Vordergrund, das Handy mit Verboten aus der Schule herauszuhalten und auf den kritischen, bewussten Umgang mit dem Handy hinzuwirken. Es sind die vertrauten Umgangweisen mit Medien aus dem Unterhaltungsbereich. Was ist darüber hinaus denkbar? Das ist eine Frage an die Mediendidaktik, die in Deutschland eine Reihe theoretischer Zugängen entwickelt hat (siehe Tulodiziecki, Herzig 2004, S. 109 ff, Kron, Sophos 2003, S. 53 ff., Sacher 2005, Kerres 2005, Diskussion weiter unten). Aus dieser mediendidaktischen Diskussion sind zwei Argumente wichtig (siehe Pachler, Bachmair, Cook 2010, S. 173 ff). Sie leiten auch den Modellversuch My Mobile, Handy im Unterricht:

- Informelles Lernen, die Kompetenz von Kindern und Jugendlichen im Mobilitätskomplex und neue habitualisierte Lernformen an die Schule heranführen und in den Unterricht einbinden. Es geht also um die didaktische Assimilation des Mobilitätskomplexes in die Schule.

- Wichtiges Mittel der didaktischen Assimilation ist, auf den Alltag der Schülerinnen und Schüler und ihre Entwicklung im Alltag einzugehen. Dieser Alltag ist mittlerweile mit dem Handy verbunden, ja sogar mit dem Handy verschmolzen. Wie lässt sich auf der Basis eines Assimilations-Modells auf die Schüler eingehen? Mit ihrem Handy und ihren Aktivitäten im Medienkonvergenzsystem bauen sie Kommunikationsbrücken und Kommunikationsketten vom Alltag in die Schule (siehe unten: Eckpunkt 2.4). Als Aufgabe kommt auf Lehrerinnen und Lehrer zu, solche Kommunikationsbrücken und Kommunikationsketten zu entdecken und an Lernsituation anzubinden. Weiterhin verlangt die Individualisierung von der Schule, bei ihren institutionalisierten Lehrprozessen auf die Entwicklung der Kinder einzugehen. Die Entwicklung der Kinder liefert den Rahmen für Lehren und Lernen (siehe unten: Eckpunkt 2.6)

Der Gedanke der kindlichen Entwicklung als Rahmen erfolgreichen Lehrens und Lernen leitet sich von Lev Vygotsky (1978 / 1930, S. 84ff, 1986/ 1934, S. 154 ff.) ab und seinem Konzept der «zones of proximal development». Der didaktische Gedanke der Kommunikationsbrücken und Kommunikationsketten basiert auf dem der «threads» im Internet; das sind Kommunikationsverläufe und Kommunikationsketten im Internet. Diana Laurillard (2007) liefert dazu das Modell. Sie sieht im Unterricht ein Gefüge von «conversational processes». Das Handy hat dabei die Aufgabe, die Kommunikationsprozesse des Alltagsleben mit denen der Schule zu verbinden. Abbild 1 bietet die Veranschaulichung von Diana Laurillards Modell der "conversational processes» (Interpretationsstrukturen des Unterrichts), in das zur Veranschaulichung das Bild eines Handy als Schnittstelle von Alltag und Schule eingefügt ist. 


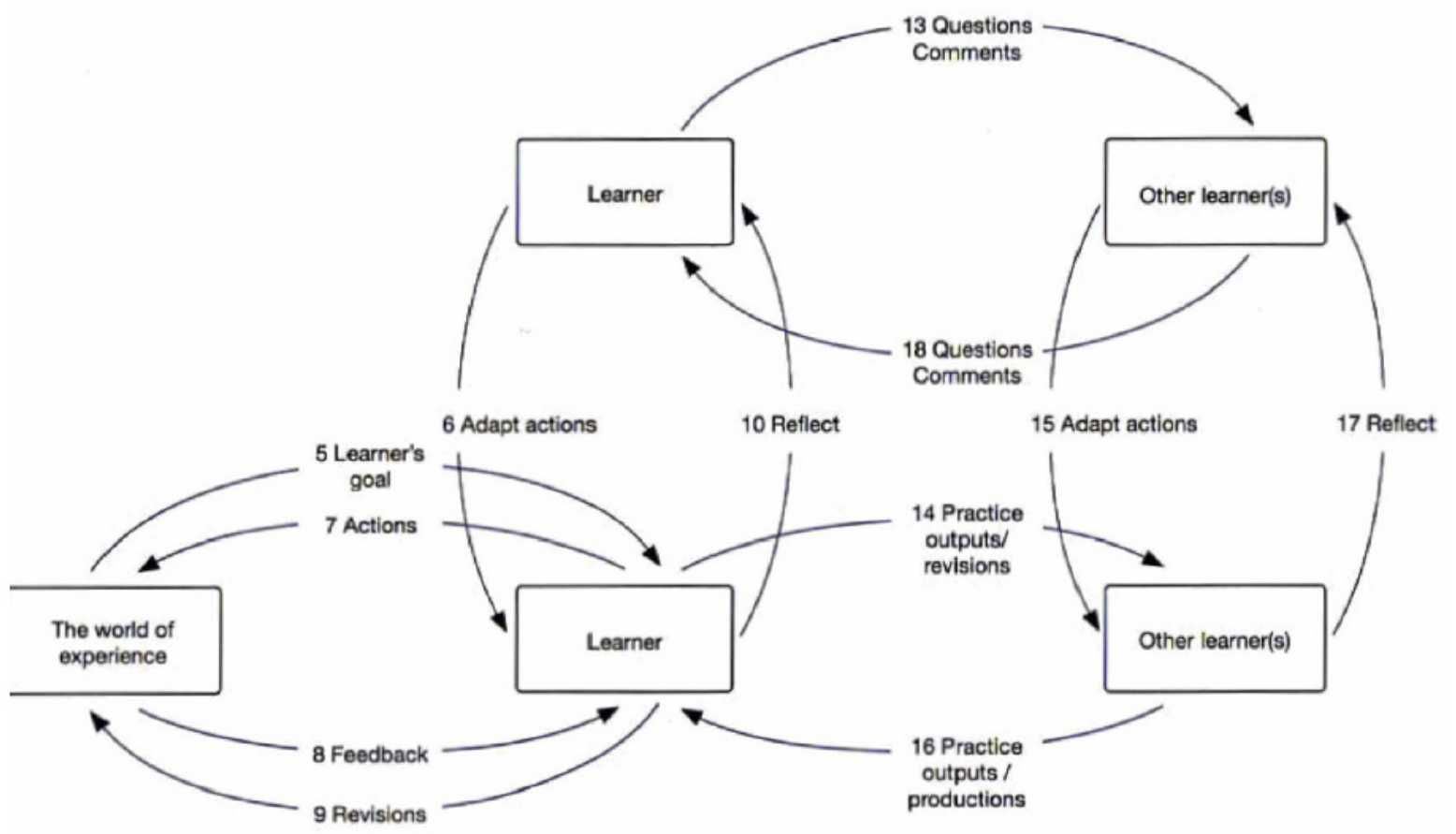

Abbildung 1: Interpretationsstrukturen des Unterrichts («conversational processes») nach Laurillard 2007, S. 171

Dies Schema der Interpretationsstrukturen des Unterrichts von Diana Laurillard (2007; S. 171) zeigt die Öffnung der Schule, die mit dem Handy gelingen kann. Die Schule gewinnt im Sinne des "Conversational Framework» (Laurillard 2007) über die Schüler/innen («Learner») den Anschluss zu deren Erfahrungswelt («The world of experience»). Dazu gehören Unterrichts- und Alltagsstrategien wie Ziele zu setzen («Learner's goal»), zu handeln («Actions»), Rückmeldungen («Feedback») und Überprüfungen («Revision»). Hierbei können Handy-Funktionen (Mobilität, individuelle Verfügbarkeit, Foto, GPS, Anschlussfähigkeit zum PC) eine eigenständige und konstitutive Rolle übernehmen.

\subsection{Handy, Kulturressourcen und Bildung: ein kulturökologischer Rahmen}

In der bisherigen Argumentation spielte der Begriff der Kultur-Ressource, Medien als Ressourcen, eine tragende Rolle. In die Didaktik kam dieser vor allem ökonomische Begriff mit der Beschreibung der Industriegesellschaft, die sich im Prozess der Globalisierung zur Wissensgesellschaft entwickelt. In dieser Denklinie und in der Linie der gesellschaftlichen Entwicklung macht es Sinn, informelles und formelles Lernen, Kompetenzen, Literalität oder Medien als Kulturressourcen zu behandeln. Zu Ressourcen gibt es die bekannten Auseinandersetzungen um Zugänge, Verfügbarkeit und Macht, so auch beim Mobilitätskomplex. Ein aktuelles 
Beispiel ist Googles Schritt aus dem Internet in das Alltagsleben, indem Google die Strassen und Häuser fotografiert. Damit gewinnt Google den Zugang zu den Handynutzer/innen, die, wo auch immer sie stehen oder gehen, per App die Informationen zu den umgebenden Konsumangeboten oder Kultureinrichtungen bekommen. Ebenso ermöglicht Street View den aktuellen Handy-Standort mit den in der Medienkonvergenz verfügbaren Informationen zu individuellen Profilen zu verknüpfen.

Warum diese Ressourcen des Mobilitätskomplexes Google überlassen und sie nicht für Teilhabe aller an Bildung ausschöpfen? Wie lässt sich jedoch diese schlichte Analogie von Ökonomie und Bildung auch pädagogisch begründen? Dazu kurz zwei Argumente, die an anderer Stelle ausführlich entwickelt wurden (siehe Bachmair 2009, S. 89 ff., 155 f.; Bachmair, Cook, Pachler 2009; Pachler, Bachmair, Cook 2010, S. 73 f, 155 ff.) Zum einen geht es um die Rolle von Kulturressourcen im Gefüge von Strukturen, Handlungs- und Persönlichkeitsformen sowie von Kulturpraktiken. Zu den Kulturpraktiken gehört u. a. die Mediennutzung im Alltag oder das formelle Lernen in der Schule. Im Moment versuchen Lehrer/innen, die Kulturpraxis der Schule aus den sich ändernden gesellschaftlichen Strukturen herauszuhalten. Dazu gehören die Verschiebungen, die mit dem Handy im Mobilitätskomplex einhergehen, wie z. B. mit von Nutzern/-innen generierte Kontexte. So ein nutzer/innengenierter Kontext ist z. B. der Bereich der Hausaufgaben auf YouTube, in dem eine riesige Menge an kommentierten Handy-Videos steht. Die London Mobile Learning Group hat diese Gefüge von Strukturen, Handlungs- und Persönlichkeitsformen und Kompetenzen (agency) und kulturellen Praktiken mit Hilfe eines Dreieckmodells veranschaulicht.

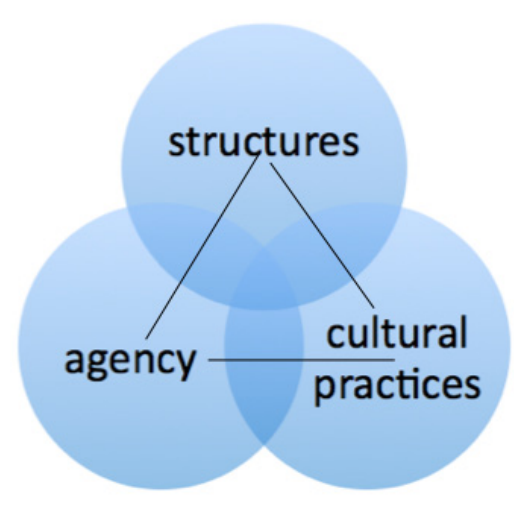

Abbildung 2: Dreiecksmodell zur Analyse der sozialkulturellen Entwicklung (Pachler, Bachmair, Cook 2010, S. 25) 
Dieses Gefüge von gesellschaftlichen, sozial-kulturellen Strukturen, Handlungskompetenzen und Persönlichkeitsformen (agency) sowie von kulturellen Praktiken kann eine Gesellschaft dem Machtspiel oder dem Machtkampf der gesellschaftlichen Kräfte überlassen. Sie kann jedoch auch versuchen, diese Ressourcen so zu verteilen, dass alle drei Bereiche sich in ihrer Eigengesetzlichkeit aufeinander bezogen entwickeln. Mit dieser Aussage: versuchen zu verteilen, kommt eine Art handelndes Subjekt in die Diskussion. Das ist natürlich ein Optimismus, der so einfach nicht funktioniert. Als Pädagogin oder Pädagoge kann frau / man versuchen, sich mit intelligenten Vorschlägen wie einem Modelversuch in die Schulentwicklung einzumischen. Mit dem zweiten Gedanken, dem der Eigengesetzlichkeit, überträgt man / frau die Auseinandersetzung der 1970er und 1989 Jahre um Natur und Energie als Ressource auf den Bereich der Kultur. So gesehen lässt sich das Handy im Mobilitätskomplex z. B. mit neuen informellen Lernformen abwägend verbinden. Dazu gehört z. B. nach Unterrichtspraktiken zu suchen, wie das Handy als Alltagsressource von Risikolernern/-innen in der Hauptschule funktioniert.

Das Stichwort dazu heisst, das Handy als Ressource im Rahmen einer Kulturökologie in eine etablierte Schulpraxis einzufügen. Einfügen in die Schulpraxis entspricht dem oben formulierten Prinzip der Assimilation.

Das zweite Argument neben der Rolle von Kulturressourcen muss sich auf Bildung beziehen. Dieses muss in dieser Aufgabenstellung, ist nicht zufällig gewählt. Optimale Lernressourcen auszuwählen und sie in der Schule einzusetzen, ist allein noch nicht hinreichend und braucht eine pädagogische Legitimation. Dazu ist der Einsatz neuer oder alter Kulturressourcen von der Entwicklung der Kinder her zu begründen und zu rechtfertigen. In der Perspektive von Bildung entwickeln sich Kinder. Sie entfalten ihre Kräfte, so Bildung im Sinne von Wilhelm von Humboldt, indem sie sich die Kulturobjekte ihrer gesellschaftlichen Welt aneignen (vgl. Bachmair 2009, S. 157 ff., S. 227). Aneignung des Handys, Aneignung des Mobilitätskomplexes ist hierzu die Schlüsselkategorie, um auch Lernen in der Schule zu begründen (vgl. Pachler, Cook, Bachmair 2010). Mit dem Gedanken der Bildung kommt der pädagogische Auftrag in die Diskussion des Handys als Lernressource. Bildung basiert auf der Aneignung kultureller Güter, die die Grundlage der kindlichen Entwicklung ist. Im Humboldtschen Sinne ist Bildung eine mannigfaltige Ganzheit. Ebenso gehört dazu, dass die Kinder oder Jugendlichen mit ihren Spuren die Welt gestalten, sie Vernunft realisieren und dass sie all dies in Freiheit tun (siehe Bachmair 2009, S. 161 ff.).

Mit dieser mehr oder weniger explizit formulierten Bildungsausrichtung (z. B. Kerres 2005, S. 227) versucht heute Didaktik, die Schule für die Entwicklung der Kinder sowie für Gestaltung und die Ganzheit von Schule und dem Leben der Kinder zu öffnen. Wenn z. B. Werner Sacher (2005) für ein «arrangiertes Lernen» eintritt, wenn er "Lernarrangements» (S. 174), "Lernsituationen und Lernumgebungen» (S. 175) als didaktische Aufgabe an Lehrer/innen und Lehrer heranträgt, dann will er damit 
«Verständigungsprozesse» (S. 179) ins «Aktgefüge des Lernens» u. a. mit «Erfahren, Erleben, Präsentieren, Anwenden und Üben, Integrieren, Festigen, Kontrollieren, Kommunizieren, Organisieren» (Modell S. 184) integrieren. All diese Prozesse sind auf die «Lebenspraxis als Bezugshorizont» ausgerichtet.

Die anglophone Diskussion stellt Lernen als Bedeutungskonstitution (meaningmaking) heraus, die subjektzentriert in den Kontexten unterschiedlicher kultureller Praktiken stattfindet (z. B. Kress 2010). Natürlich ist nicht jede Form, mit den Kinder Sachverhalten, Ereignissen, Zeichen oder Medien Bedeutung zu verleihen und sich Bedeutung anzueignen, auch schon Lernen. Beim Lernen steht unter anderem im Vordergrund, Kenntnisse gezielt abzurufen und beabsichtig zu verwenden. Bedeutung entsteht jedoch immer in Rahmen von Kontexten. Die Schule stellt hierzu einen standardisierten Kontext zu Verfügung. Es gibt aber viele Kontexte, in denen Kinder oder Jugendliche Wissen generiert, indem sie eigene Bedeutungen von Sachverhalten entwickeln. Dieser Zusammenhang von Bedeutung und Kontext fällt didaktisch gesehen unter das Stichwort des Situierten Lernens (Lave, Wenger 1991; Tulodziecki, Herzig 2004, S. 146 ff.), das heute auch als partizipatives Lernen angelegt ist (siehe Patten et al. 2006). Situiertes Lernen kommt nicht als irgend ein edles Ziel in die Didaktik, sondern weil es den Strukturen der heutigen Lebenswelt und dem Habitus von Risikolernen entspricht.

\subsection{Das Papier «Eckpunkte einer Didaktik des mobilen Lernens»}

Wie lassen sich diese komplexen Überlegungen zur sozial-kulturellen Entwicklung und den daraus erwachsenden didaktischen Aufgaben in den Schulalltag umsetzen? Der Schulversuch MyMobile - Handy im Unterricht von Medien+Bildung.com in Rheinland-Pfalz hat 2009 und 2010 sechs Unterrichtseinheiten in Regelschule durchgeführt. Eine wesentlich Aufgabe war, den oben skizzierten Bezugsrahmen zu operationalisieren, was mit dem Papier «Eckpunkte einer Didaktik des mobilen Lernens» geschah (Text des Papiers siehe weiter unten). Der Entwicklung dieses Eckpunkte-Papiers ging in der London Mobile Learning Group die Diskussion von Didactic parameters for analysing and planning within the mobile space of convergence and learning (Pachler, Bachmair, Cook 2010, S. 297 ff.) voraus. Theoretisch systematisch gesehen, verbinden die didaktischen Parameter das schulische Lernen im Raum der Schule mit dem mobilen, individuellen und konvergenten Raum des Mobilitätskomplexes. Das Modell der Parameter beschreibt diesen mobilen, individuellen und konvergenten Raum im Verhältnis zur Schule: 
Parameter 1: Lehr- und Lernform

Pol: Schulpraxis - Pol: Alltagsnutzung des Handys

Parameter 2: Verhältnis zum Lerngegenstand

Pol: mimetische Reproduktion - Pol: subjektive Rekonstruktion

Parameter 3: institutioneller Schwerpunkt des Lernens

Pol: Schulcurriculum - Pol: subjektive Kompetenz von Schülern als naive

Experten

Parameter 4: Medien und Darstellungsformen

Pol: Isoliert, linear (mono-medial, mono-modal)- Pol: konvergent

Die Bewertung der Ergebnisse des Modellversuchs im abschliessenden 4. Teil basiert auf diesen didaktischen Parameter.

Die folgenden Eckpunkte einer Didaktik des mobilen Lernens bilden das operationale Bindeglied zwischen den Parametern eines mobilen, individuellen und konvergenten Raumes, in denen mobile Endgeräte vorhanden sind, und der curricularen Nutzung des Handys in der Schule. Wie ist das Eckpunkte-Papier aufgebaut? Es operationalisiert als erstes die Ziele des Handy-Einsatze und gibt dafür eine knappe Begründung.

\section{Eckpunkte einer Didaktik des mobilen Lernens ${ }^{4}$}

Ziel des Handy-Einsatzes in der Schule ist, den Alltag der Kinder und Jugendlichen mit seiner typischen Medienkultur in die Lernformen der Schule zu integrieren. Dazu gehört auch die ausserhalb der Schule erworbene Kompetenz im Umgang mit den vernetzten digitalen Medien, die vom Computer bis zum Internet (Medienkonvergenz) reicht. Das Handy soll zudem den Lernerfolg in der Schule mit neuen Lernformen fördern. Neue Lernformen sollen auch Schüler/innen und Schüler mit Distanz zu Schule an den Lernerfolg der Schule heranführen.

1. Warum? Mobiles Lernen ist die didaktische Antwort auf die veränderte Medien- und Lernkultur vieler Kinder und Jugendlicher

1.1 Die neue dominante Medienkultur des Alltags: individualisiert, mobil und konvergent

Mobiles Lernen ist eine didaktische Antwort auf den aktuellen Individualisierungsschub mit der mobilen und vernetzten Massenkommunikation. Eine spezifische Didaktik mobilen Lernens reagiert auf den aktuellen sozialkulturellen und technologischen Wandel, bei dem das alltägliche Handy den allgegenwärtige und individuellen Zugang zu Kommunikation, Unterhaltung, Konsum, Internet, Medienangeboten oder auch zu Wissensarchiven liefert.

4 Konzeption und Copyright der Eckpunkt einer Didaktik des mobilen Lernens einschliesslich der Didaktischen Eckpunkte und der Eckpunkte zu den Nutzungsfunktionen des Handys: Ben Bachmair www.ben-Bachmair.de 2010 
Mit dem Einsatz des Handys im Rahmen von Unterricht und curricularem Lernen trägt Schule der immer bedeutsamer werdenden Alltagsfunktion des Handys als Multimediagerät Rechnung. Wichtig sind auch die Funktionen des Handys als Zugangsmedium zum Web 2.0 und zu den verschiedenen sozialkulturellen Milieus.

1.2 Zunehmende Bedeutung informellen Lernens und abnehmende Reichweite schulischen Lernens

Mobiles Lernen ist zudem eine didaktische Antwort auf die wachsende Bedeutung des informellen Lernens und des «Überall-Lernens» ausserhalb der Schule sowie auf die hohe Zahl bildungsferner Risikolerner in der Schule. Gerade für bildungsferne Schüler/innen und Schüler lässt sich mit dem Handy das informelle Lernen ihres Alltags in den Schulunterricht integrieren. Das Handy eröffnet Chancen für die Assimilation von informellem und formalem Lernen, indem es die vielfältigen Themen und unterschiedlichen Erlebnisweisen von Schüler/innen und Schülern einer fragmentierten Gesellschaft unterstützt. Ziel ist es, den schulischen Lernerfolg zu fördern.

Den allgemeinen Zielen und der Begründung des Mobilen Lernens folgen zwei Listen mit konkreten Eckpunkten. Das ist die Liste der Eckpunkte zur Didaktik ( 2 . Wie? Die Strukturmerkmale des mobilen Lernen») und die Liste der Eckpunkte zu den technischen Funktionen des Handys («3. Womit? Die multimediale Funktionsvielfalt des Handys als mobiler Mini-Computer und Schnittstelle der Medienkonvergenz»). Die Eckpunkte Didaktik und die Eckpunkte Handy-Anwendungen leiteten die Vorbereitung und die Diskussion der sechs Unterrichtseinheiten des Modellversuchs ${ }^{5}$. Die folgenden Teile 2 und 3 des Artikels geben einen kappen Überblick über die Anwendung der Eckpunkte in zwei dieser Unterrichtseinheiten:

\section{Unterrichteinheit «Konstruieren und Messen von Kreisen und Winkeln» im Mathematikunterricht eines 6. Schuljahres eines Gymnasiums}

Elisabeth Langgässer Gymnasium, Alzey

Zeitrahmen: 4 Wochen, mit jeweils 4 Stunden

28 Schüler/innen, 12 Jahre alt

Klassenlehrer, Medienpädagogin, Praktikantin

Ausstattung: Klassenzimmer mit Tafel und interaktivem Whiteboard, Schul-

PC, Internet, 10 Projekthandys Nokia N85, weitere Schülerhandys

5 Als einfache Form der Evaluierung der Unterrichtseinheiten gab es eine Schülerbefragung, die jedoch noch nicht abschliessend ausgewertet ist. 
Unterrichtseinheit «Buchstaben und Laute erkennen, bestimmen und lokalisieren» im Erstlese- und Schreibunterricht im 1. Schuljahr einer Grundschule.

Grundschule Luitpoldschule, Ludwigshafen

Zeitrahmen: 5 Wochen, insgesamt 9 Doppelstunden

26 Schüler/innen, 6-7 Jahre alt

Klassenlehrerin, Medienpädagogin, Praktikantin

Ausstattung: Klassenzimmer mit Tafel, 2 Schul-PCs, Beamer, 19 Projekthandys Nokia N85

\section{Die didaktischen Strukturmerkmale des mobilen Lernens - sechs Eckpunkte für die Planung und Analyse des Unterrichts mit dem Handy}

Die obige Einleitung des Eckpunkte-Papiers orientiert sich an den aktuellen sozialkulturellen Veränderungen, die nicht nur zu neuen medialen Formen, sondern auch zu neuen Lernformen ausserhalb der Schule führen. Deshalb deckt der erste didaktische Eckpunkt das informelle Lernen ab. Informelles Lernen ist zwar hoch relevant für den ausserschulischen Bereich, trifft jedoch in der Schulpraxis eher auf einen blinden Fleck. Obwohl die Entwicklung hin zum informellen Lernen bewusst beim Schulversuch MyMobile - Handy im Unterricht bedacht wurde, so war es doch schwer, informelles Lernen in der vertrauten Schulpraxis zu entdecken oder gar zu ermöglichen. Erfolgreich war hingegen die für die Unterrichtsplanung leitende Idee, Episoden situierten Lernens mit Hilfe des Handys in den lehrer/ innengeleiteten Unterricht einzufügen. Beim situierten Lernen bietet die Lehrerin oder der Lehrer Situation an, in denen Schüler/innen sich mit Themen auseinandersetzen, in denen sie Wissen selber entwickeln oder selber abrufen. In den revidierten Didaktischen Eckpunkten des mobilen Lernens wird deshalb auch das Schlüsselelement des didaktischen Designs (Brown 1992), das ist das situierte Lernen, am Anfang stehen. In der Unterrichtseinheit der Grundschule im Erstlese- und Schreibunterricht der 1. Klasse gibt es situiertes Lernen in der Form von Lern-Stationen. In den Lern-Stationen kneten die Kinder Buchstaben mit Plastilin, schreiben sie in Sand oder suchen und fotografieren Wörter mit dem Handy, in denen der aktuelle Buchstabe enthalten ist. Im Mathematikunterricht der 6 . Klasse fotografieren die Schüler/innen z. B. zu Hause und als Hausaufgabe Winkel, die sie in ihrer Wohnung oder in ihrer Umgebung finden.

Die Beispiele für die folgende Erläuterung der sechs didaktischen Eckpunkte stammen aus zwei der sechs Unterrichtseinheiten des Schulversuchs MyMobile Handy im Unterricht, und zwar aus dem Erstlese- und Erstschreibunterricht der Grundschule Luitpoldschule in Ludwigshafen und aus dem Mathematikunterricht des Elisabeth-Langgässer-Gymnasiums in Alzey. 
Didaktischer Eckpunkt 2.1: Mit dem Handy informelles Lernen in die Schule integrieren

Das Alltagsmedium Handy bietet die Möglichkeit, informelles Lernen und Wissen des Alltags in die Schule einzubinden. Es kann als Schnittstelle zwischen der Kinder- bzw. Jugendkultur, dem Alltagsleben und dem gezielten Lernen im Unterricht funktionieren.

\section{Fotosafari im Erstlese- und Schreibunterricht}

Ein Beispiel dazu ist die Foto-Safari mit dem Handy, bei der sich die Kinder in der Schule aber auch zu Hause auf Entdeckungsreise nach den Elefantenwörtern (zusammengesetzte Nomenwörter) begeben (vgl. auch Teil 3, Eckpunkt 3.4). Das passt zum Lehrplan Deutsch, der vorschreibt, dass Kinder über Sprache nachdenken und in diesem Zusammenhang Begriffe klären, Wortbedeutungen erschliessen sowie Regelhaftigkeit erkennen sollen (Rahmenplan Grundschule, Teilrahmenplan Deutsch Rheinland-Pfalz, 2005, Seite 11). Mehrere Kinder präsentieren ihre mitgebrachten Fotos im Gesprächskreis. Gemeinsam überlegen die Kinder, wie viele und welche Wörter sich in den grossen Elefantenwörtern verstecken, z. B. Osterhase, Aufgabenheft, Abfallwirtschaftsgesellschaft, Müllheizkraftwerk, Schulranzen.

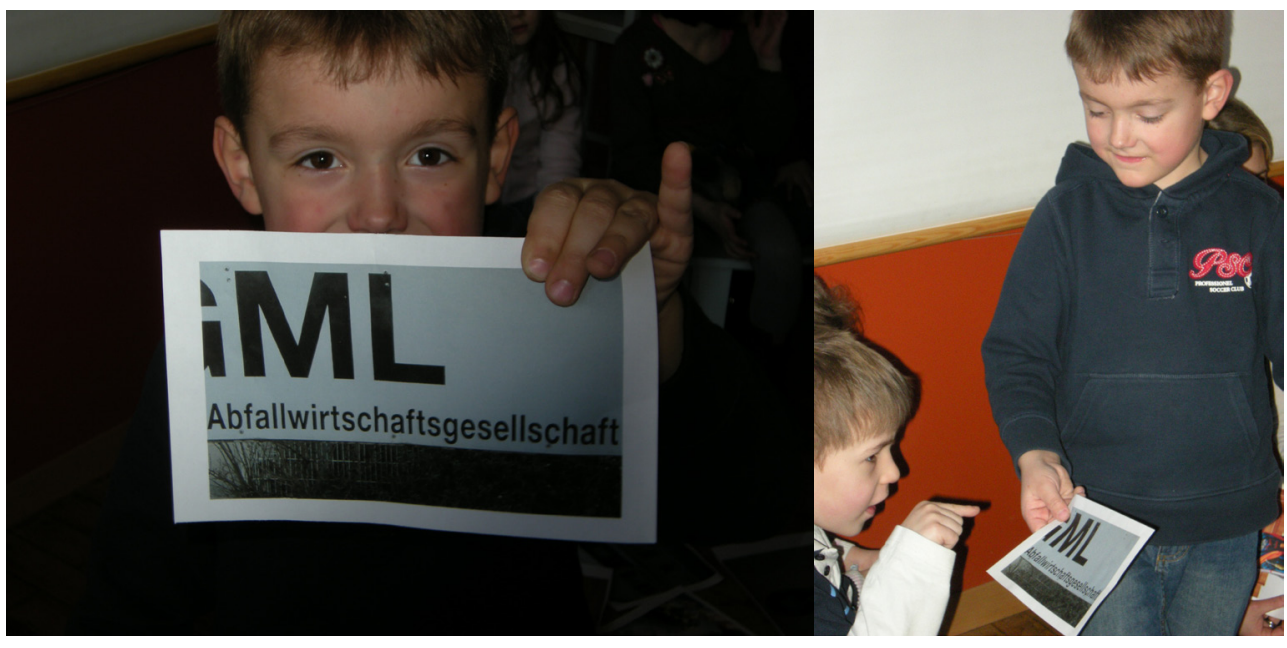

Abbildung 3: Präsentation der Hausaufgabe in der Grundschule

Ein Junge präsentiert stolz seine mitgebrachten Fotos, die aus mehreren Wörtern bestehen: Müllheizkraftwerk, Abfallwirtschaftsgesellschaft. Er erzählt, dass er die Wörter bei einem Ausflug mit seinem Vater zur Müllverbrennungsanlage gefunden hat. Diese Hausaufgabe mit dem Handy ist eine noch recht schultypische Form, informelles Lernen des Alltags in die Schule zu integrieren. Das Handy liefert den Kindern die Möglichkeit, etwas für sie Bedeutsames in ihrer Lebenswelt zu fotografieren, die Fotos mitzubringen und zu präsentieren. 
Im Mathematikunterricht der 6. Klasse eines Gymnasiums öffnet die Hausaufgabe, Winkel mit dem Handy zu fotografieren, das Tor zum informellen Lernen. Ein 12-jähriger Junge nimmt mit seinem Handy Fotos mit unklaren, schwer definierbaren Winkeln auf, verknüpft die Fotos zu einem Video und unterlegt dieses Video mit einem Popsong aus Pokémon (siehe dazu Eckpunkt 2.6 unten). Der unklare Winkel ergibt sich daraus, wie der Junge die Kameraperspektive nutzt. Später macht der Lehrer die Beziehung von Winkel, Abbild und Perspektive zum Thema einer Lern-Episode. Winkel und Abbildungsperspektive kommen auf diesem informellen Weg in den Unterricht. Die informelle Mathematik von Pokémon bleibt dagegen weiter ausserhalb der Schule und im Verborgenen der Massenkommunikation. Bei Pokémon spielen zwar Winkel keine Rolle; ein entscheidendes Thema von Pokémon ist dagegen jedoch die Leistungsmessung in Wettbewerben. Messen bei Wettkämpfen und Leistungsvergleichen sowie die Darstellung von Messergebnissen ist für Jungen bei Pokémon ein relevantes Thema. Eine Gruppe von Mädchen stellt eine andere Beziehung zur Massenkommunikation her, indem sie eine von ihnen gespielte Talkshow mit dem Handy aufnehmen. In dieser Experten/-innenrunde versprachlichen sie spielend ihre Ergebnisse zur Handynutzung im Unterricht und mit Bluetooth.

\section{Didaktischer Eckpunkt 2.2: Mit dem Handy Episoden situierten Lernens schaffen}

Das Handy und seine Nutzungsmöglichkeiten bieten neue Formen des situierten Lernens. So lässt sich der vom Lehrer geleitete Unterricht mit Episoden handygestützter Schüleraktivitäten verbinden. Bildlich formuliert, erweitern die Lern-Plätze der Schüler (= Episoden des situierten Lernens) die Lern-Strasse des Lehrers (= Phasen des lehrergeleiteten Lernens).

Ende der 1980er-Jahre entwickelte sich in der US-amerikanischen Pädagogik mit dem situierten Lernen (Lave, Wenger 1991) ein theoretisches Modell als Gegenentwurf zum Instruktionslernen, also dem wiederholenden Erlernen vorgegebener Lerninhalte. Ausgangspunkt für diesen Gegenentwurf des situierten Lernens war eine für Sprechtheorie und Semiotik damals selbstverständliche Überlegung, dass Bedeutungen nicht transportiert, sondern immer in Abhängigkeit von der Situation, in der die Menschen denken, handeln und kommunizieren, entstehen. Die Menschen stellen also in ihrer Kultursituation Bedeutung her. Lernen ist eine kulturelle Form der Herstellung von Bedeutungen, in der sich Kinder oder Jugendliche je nach der dominierenden Situation etwas aneignen, weil sie selber Objekten, Emotionen, Vorgängen, Gesprächspartnern und deren Aussagen oder Texten und Medien Bedeutung verleihen. Sie lernen also in der Schule als Schüler; sie lernen vor dem Bildschirm mit Unterhaltungsanspruch oder in einer Werkstatt als Lehr- 
ling, weil sie in diesen Situationen aus den vorgegebenen Inhalten und Tätigkeiten für sich Bedeutsames schaffen (Brown et al. 1989, Lave, Wenger 1991, Hanks 1990). In deutschen Schulen dominiert der lehrergeleitete Unterricht, der sich jedoch mit Episoden situierten Lernens bereichern lässt. Das Handy als Instrument der Schüler/innen und Schüler eignet sich, Episoden situierten Lernens in den lehrergeleiteten Unterricht einzufügen. Bei der Erprobung dieses didaktischen Eckpunktes war folgende Formel hilfreich: Der Lehrer oder die Lehrerin führt die Schüler/innen entlang einer Lern-Strasse, die sich an den Lernzielen und Lernthemen orientiert. Lehrerin oder Lehrer bietet Schülern/-innen Lern-Plätze (= Episoden). Auf diesen Lern-Plätzen (Episoden des situierten Lernens) beschäftigen sich die Schüler/innen eigenständig mit dem Lernthema.

Im Schulversuch MyMobile - Handy im Unterricht war zusätzlich neben der Lehrerin oder dem Lehrer auch noch eine Medienpädagogin oder ein Medienpädagoge mit für Planung und Realisierung des Unterrichts mit Lern-Strasse (lehrer/innengeleiteter Unterricht) und Lern-Plätzen (Episoden situierten Lernens) zuständig. Die Medienpädagoginnen und Medienpädagogen konzentrierten sich vor allem auf die Handy gestützten Episoden situierten Lernens.

\section{Handy-Station beim Erstlesen und Erstschreiben in der Grundschule}

Beim Erstlesen und Erstschreiben in einer 1. Klasse Grundschule bietet die Lehrerin sowohl bei der Einführung eines neuen Buchstabens oder Lauts als auch in der Phase der Vertiefung situiertes Lernen in der Form des Stationen-Lernens an. Im Sinne der Metapher von Lern-Strassen und Lern-Plätzen (Episoden situierten Lernens) sind Lern-Stationen mehrere Plätze, die die Lehrerin den Kindern öffnet. Die Kinder können z. B. einen Buchstaben kneten oder, in einer anderen Lern-Station, Gegenstände, die den Buchstaben enthalten, mit ihrem Handy suchen und fotografieren. Pro Woche wird ein neuer Buchstabe oder ein neuer Laut eingeführt und vertieft, wobei die Lehrerin mit zwei Typen von Lern-Stationen arbeitet. Auf die Einführungsphase mit sechs Lern-Stationen folgen drei Lern-Stationen zur Übung und Vertiefung (siehe Abbildung 4). Dabei arbeiten die Kinder auch in einer Handy-Station, die einen der möglichen Lern-Plätze in der Lern-Strasse des lehrerinnengeleiteten Unterrichts eröffnet. 


\section{Handy-Station bei der Einführung eines neuen Buchstabens od. Lautes}

\begin{abstract}
Handy-Station bei der Übung und Vertiefung des Sprachgebrauchs
\end{abstract}

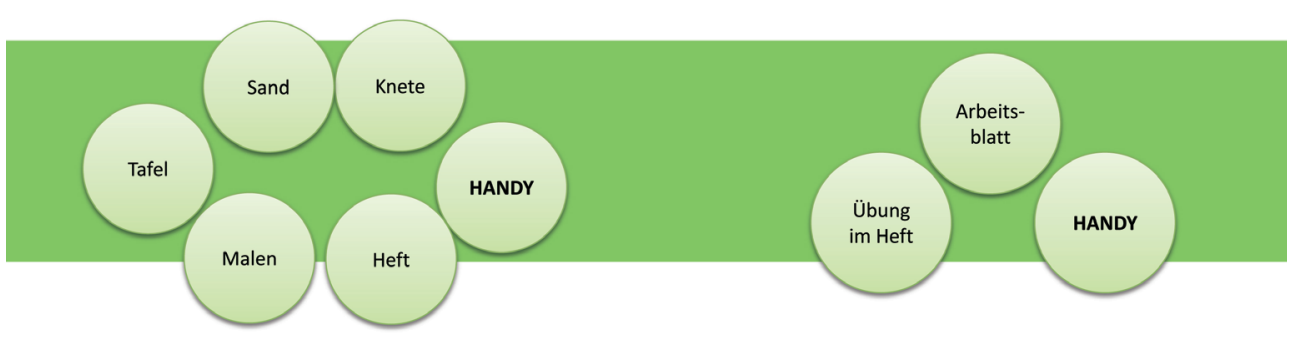

Abbildung 4: Situiertes Lernen als Handy-Station in der Grundschule

Bei der Einführung eines neuen Buchstabens oder Lautes gibt es fünf unterschiedliche Lern-Stationen, an denen die Kinder den Laut bzw. die Buchstaben an die Tafel schreiben, ihn kneten, in Sand schreiben, malen und Übungen im Arbeitsheft machen. Mit dieser Form des situierten Lernens in Stationen sind die Kinder nach einem halben Schuljahr vertraut. Neu für sie ist das Handy als sechste LernStation (siehe Abbildung 4). In allen Lern-Stationen arbeiten die Kinder offen und selbstständig, ohne Direktiven der Lehrerin. Die Kinder entscheiden selbst, wann und in welcher Station sie arbeiten. Sie nutzen jedoch alle Lern-Stationen. Sie beschäftigen sich also selbstständig, nach ihren Vorlieben und in ihrem Arbeitstempo mit dem jeweiligen Lernthema. Bei der Handy-Station in der Einführungsphase eines neuen Buchstabens oder Lauts gehen die Kinder z. B. in Gruppen aus dem Klassenzimmer und nehmen im Schulgebäude oder auf dem Schulhof mit dem Handy Fotos auf. Ebenso nutzen sie die Audiofunktion des Handys und sprechen die Wörter, die sie zu den Lauten und Buchstaben gefunden haben, auf ihr Handy. Dabei begleitet sie die Medienpädagogin. Die Begleitung ist vor allem notwendig, wenn die Schüler/innengruppe in der Schulumgebung nach Objekten wie z. B. einer Kirche sucht, die den aktuell wichtigen Laut - in diesem Fall das «ch» - im zugehörigen Wort enthält. Pro Doppelstunde und bei 23 Kindern bleibt für die Handy-Station ca. 15-20 Minuten.

In der Übungs- und Vertiefungsphase bietet das Handy eine von drei Lern-Stationen. In dieser zweiten Unterrichtsphase gibt es zwei Lern-Stationen innerhalb sowie die Handy-Station ausserhalb des Klassenzimmers. Im Klassenzimmer bearbeiten die Kinder Übungen in ihren Arbeitsheften, lösen Aufgaben in den Arbeits- 
blättern, die die Lehrerin aus den Fotos der Kinder erstellt hat oder recherchieren draussen wieder mit der Foto- und Audiofunktion des Handys nach Buchstaben und Lauten.

In den fünf Unterrichtswochen mit jeweils zwei Doppelstunden gab es sechs Episoden situierten Lernens, in denen das Handy eine tragende Rolle als Lern-Station spielte:

Episode 1: Namensbilder erstellen. Schüler/innen fotografieren sich gegenseitig. Die Kinder lernen die Fotofunktion des Handys kennen.

Episode 2: Auf Spurensuche nach dem «sch». Kinder fotografieren Gegenständen und Schriftzügen, in denen sich der Laut «sch» befindet. Sie sprechen diese Wörter aufs Handy (Audiofunktion).

Episode 3: Foto-Safari, Elefantenwörtern entdecken und fotografieren. Die Kinder kleben Fotos zusammen, die jetzt zusammengesetzte Nomenwörter enthalten.

Episode 4: Mit dem Z-Interview das z in mehreren Sprachen oder im Dialekt entdecken. Die Kinder nutzen die Audiofunktion bei einem Interview.

Episode 5: Lischt an oder Licht aus? Die Audiofunktion des Handys zur Kontrolle der Aussprache verwenden.

Episode 6: Auf Spurensuche nach dem sp. Die Kinder gestalten eine Bildergeschichte. Die Foto- sowie Audiofunktion zum Sammeln von Gegenständen oder Schriftzügen nutzen, in denen sich ein sp befindet. Aus eigenen HandyFotos Bildergeschichten zum sp zusammenstellen.

\section{Handygestützte Episoden des situierten Lernens}

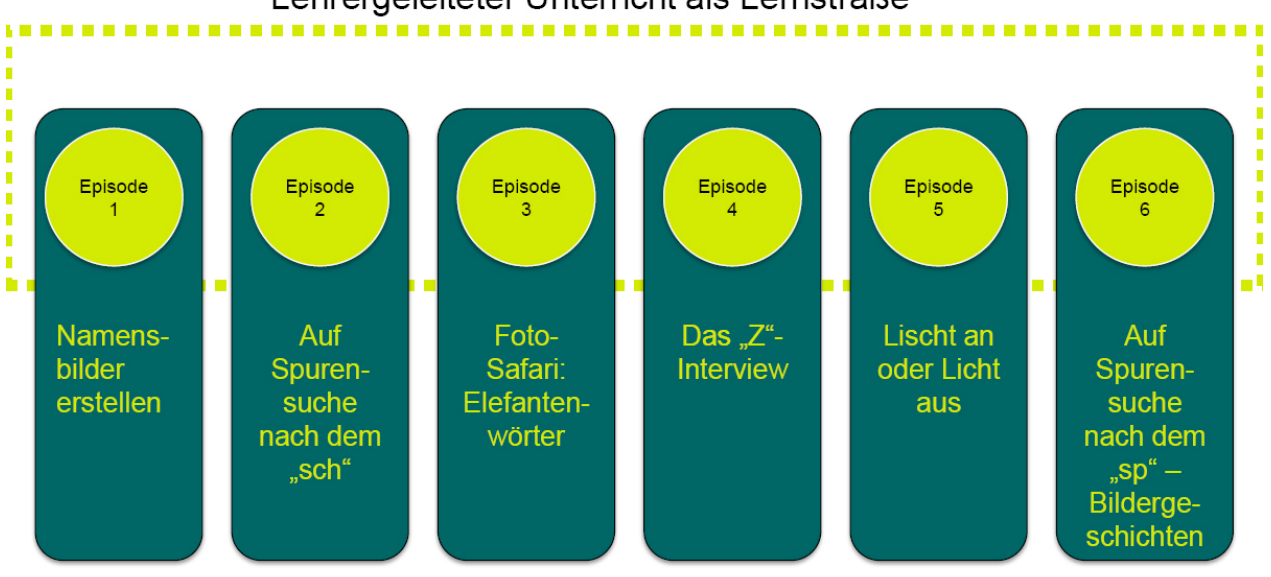

Abbildung 5: Überblick über die Episoden Situierten Lernens in der 1. Klasse der Grundschule. Diese Episoden mit dem Handy gehören zu einer Lern-Station neben anderen Lern-Stationen. 


\section{Mathematik in der 6. Klasse eines Gymnasiums}

Im Mathematikunterricht der 6. Klasse eines Gymnasiums über Kugel, Kreis und Winkel gibt es nicht wie im Grundschulunterricht parallele Episoden situierten Lernens mit dem Handy. Stattdessen folgen in der Unterrichtseinheit sechs LernEpisoden mit dem Handy aufeinander.

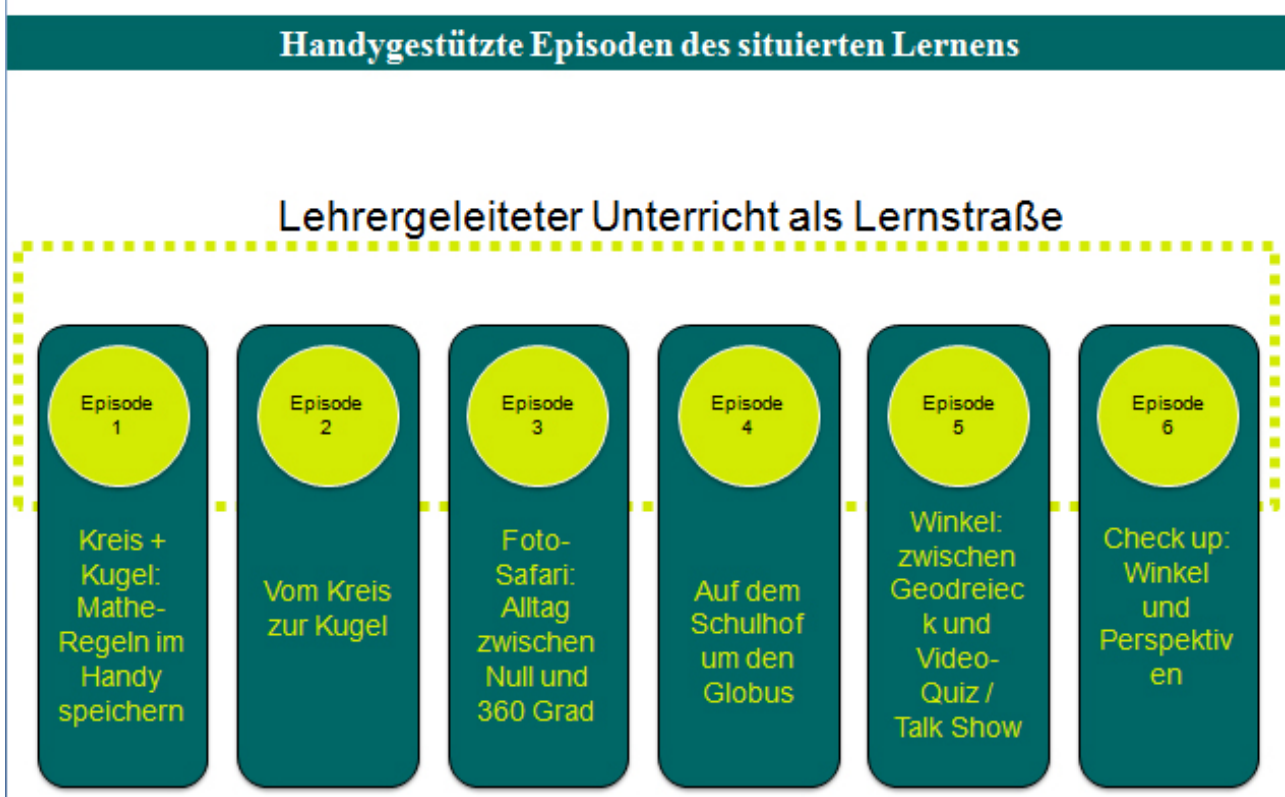

Abbildung 6: Abfolge der Episoden situieren lernens mit dem Handy in einer MathematikUnterrichtseinheit

Lernthema in Episode 1 ist der Unterschied zwischen Kreis und Kugel. Schüler/ innen und Schüler setzen dazu das Handy das erste Mal ein; sie speichern mit der Fotofunktion die entsprechenden Regeln auf ihrem Handy. Dazu fotografieren sie das Tafelbild für ihr eigenes Regelheft. In Episode 2 geht es um die Erweiterung dieser Regeln und um Begriffe wie Radius, Durchmesser und Umfang. Hier kommt erneut die Handy-Fotokamera zum Einsatz, um Regeln, Begriffe und Bilder für das eigene Arbeitsheft festzuhalten. Die Hausaufgabe führt dann zu Episode 3. Zunächst nutzen die Schüler/innen die Fotofunktion des Handys, diesmal ausserhalb des Unterrichts. Die Schüler/innen fotografieren wahlweise Kugeln, Kreise und Winkel in ihrer Umgebung. Zur Besprechung dieser Hausaufgabe sichtet die Klasse zusammen mit dem Lehrer die Fotos auf dem interaktiven Whiteboard. Dann schätzen und vermessen sie die Winkel auf dem Whiteboard und im Heft. Ein Videofilm aus der Hausaufgabe wirft Fragen auf und wird danach in Episoden 6 weiter diskutiert. In Episode 4 nutzt die Klasse die GPS-Funktion des Handys um Kugelkoordinaten im Alltag zu entdecken. Episode 5 vertieft die bisherigen 
Kenntnisse. Dazu setzen die Schüler/innen die Videofunktion ein, um ein Quiz zu Winkeln zu dokumentieren. In der 6. Episoden fotografieren sie Winkel an einem Modell und bewerten sie in Bezug auf die jeweilige Aufnahmeperspektive.

\section{Didaktischer Eckpunkt 2.3: Mit dem Handy Lern- und Medienkontexte generieren}

Das Handy ist ein Instrument, mit dem Schüler/innen und Lehrer/innen neue Lernkontexte schaffen. Diese Lernkontexte entstehen an der Schnittstelle der Medienkonvergenz von Internet, Unterhaltungsmedien der Lebenswelt und der Schule. Die mit dem Handy generierten Kontexte sind weit über die Schule hinausgreifende Situationen. In diesen Situationen lernen Schüler/ innen, indem sie selber ihr Wissen schaffen und Wissen nicht nur übernehmen. Mit dem Handy generierte Kontexte sind Gelegenheiten zum situierten Lernen, die die Lern-Strassen der Schule zu Lern-Plätzen erweitern. Das Handy verbindet diese Lern-Plätze mit der vernetzten Medienwelt.

\section{Geocachen, die Ausweitung des Lernkontextes über die Klassenräume hinaus in die neue digitale Welt des Messens}

Im Mathematik-Unterricht der 6. Klasse des Gymnasiums geht es um das Lernthema Kreis, Kugel, Kugelkoordinaten und Winkel. Der Lehrer bietet den Schüler/ innen und Schülern die Episode Geocachen, in der sie nicht nur Winkel praktisch erkunden, sondern darüber hinaus ihre Schule mit den digitalen Landkarten der Satellitenortung verbinden (vgl. Teil 3, Eckpunkt 3.3). Das Handy als Instrument der Schüler/innen und Schüler, dockt' die Schule an die digitalen geographischen Netze und GPS an. Offensichtlich ist, dass die Schüler/innen und Schüler das Klassenzimmer verlassen und auf dem Schulhof mit ihrem Handy navigieren. Zum Lernkontext Klassenzimmer kommt der Schulhof als Lernkontext hinzu. Der Lernkontext kann sich auch noch um den Raum der Stadt erweitern, indem die Schülerinnen und Schüler die Koordinaten markanter Punkte ihrer Stadt vermessen und dazu gezielt die Schule verlassen.

Zum Vorgehen: Mit einem Globus auf dem Schreibtisch eröffnet der Lehrer das Thema. Das Whiteboard ermöglicht dann, auch ein Bild vom 50. Breitengrad im 35 km entfernten Mainz einzubeziehen. Die Schüler/innen schätzen die Koordinaten der Schule und «fangen» diese per GPS auf dem Schulhof ein. Über GPS können die Schüler/innen ihre Standortkoordinaten selbst herausfinden und am interaktiven Whiteboard mit Google Earth im Internet überprüfen. Medienkonvergenz, Lebenswelt und Schule verzahnen sich in dieser Episode. 

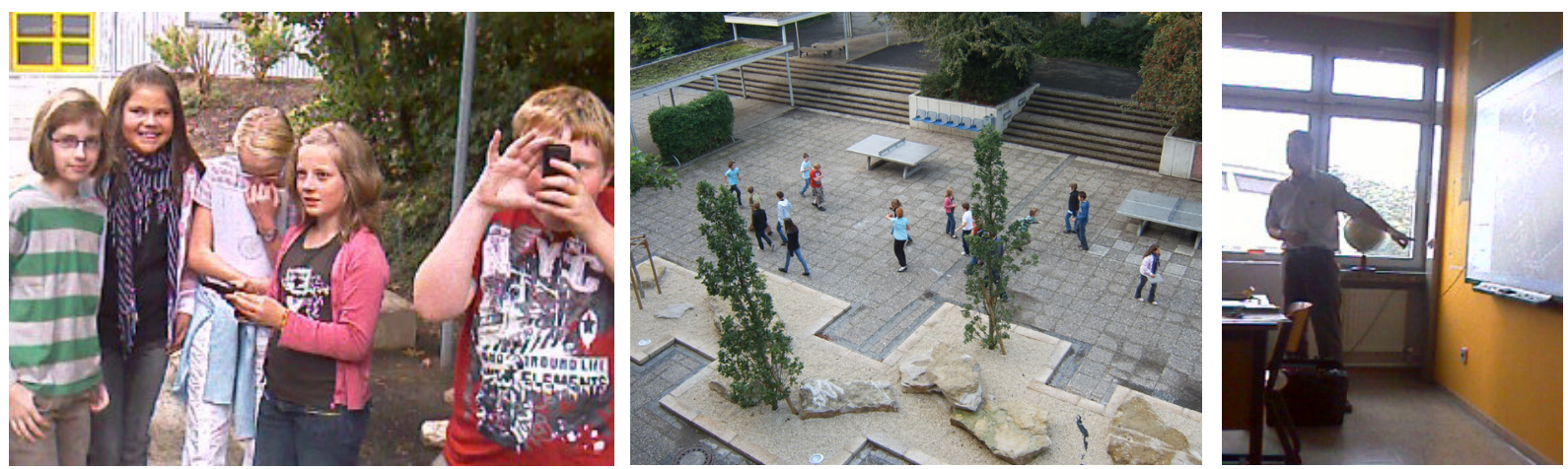

Abbildung 7: Mit dem Handy den Lernkontext vom Klassenzimmer in den Schulhof und zu Google Earth erweitern (Episode 4)

\section{Didaktischer Eckpunkt 2.4: Mit dem Handy Kommunikationsbrücken und Kommunikationsketten schaffen}

Das Handy und seine Nutzungsmöglichkeiten bieten Kommunikationsbrücken und Kommunikationsketten zwischen Alltag und Schule. Kommunikationsbrücken und Kommunikationsketten sind Verbindungslinien zwischen dem richtigen Leben ausserhalb der Schule und dem schulischen Lernen.

\section{Mathematik im Alltag - Basiswissen «Winkel schätzen und benennen»}

Die Hausaufgabe ist eine einfache Methode, um mit dem Handy eine Kommunikationsbrücke zwischen dem Unterricht in der Schule und der Lebenswelt der Schülerinnen und Schüler zu schlagen. Konkret geht es darum, Winkel zu schätzen und zu benennen. Die Schüler/innen bekommen den Auftrag, verschiedene Winkel zu fotografieren oder ein Handy-Video zu erstellen. Ein Schüler bringt Fotos mit rechten Winkeln aus seinem Wohnzimmer mit, ein anderer Winkel der Dachrinne aus seinem Garten. Die persönliche Lebenswelt der Schülerinnen und Schüler kam damit in den Blick des Unterrichts. Der Stolz des betreffenden Schülers ihre Welt in die Schule hereinzunehmen, war dabei spürbar. In diese Handy-Hausaufgaben mischen die Kinder auch ihre persönlichen Themen. So unterfüttert ein Schüler sein Winkel-Video mit einem Popsong von Pokémon, was nur auf den ersten Blick nicht zu Mathematik gehört. Schon ein genauer Blick in die Logik von Pokémon genügt, wie oben schon gesagt, um dort das Thema Leistungsmessung mit Zahlen wahrzunehmen. Das ist Teil eines geschlechtspezifischen Themas von Jungen, das etwas mit der Motivation für den Mathematikunterricht zu tun hat. Diese Motivationsbrücke zwischen Alltag und Schulunterricht liesse sich vom Lehrer eventuell nutzen. 


\section{Didaktischer Eckpunkt 2.5: Mit dem Handy die Schülerinnen und Schüler als Experten ihres Alltagslebens in der Schule individuell aktiv werden las- sen}

Im Prozess der Individualisierung und Fragmentierung unserer Gesellschaft werden Schülerinnen und Schüler zu vielfältigen Alltagsexperten. Dabei spielt das ständig verfügbare Handy eine wichtige Rolle. Die Nutzung des Handys auf den Lern-Strassen des geleiteten Lernens und in den Episoden des situierten Lernens (Lern-Plätze) unterstützt, die vielfältigen Handlungsund Lernmuster der Schülerinnen und Schüler auch für den Lernerfolg in der Schule fruchtbar zu machen.

\section{Experten/-inneneninterviews und Spurensuche in der Grundschule}

Im Lese- und Schreibunterricht der Grundschule spielt der regionale Dialekt und die nicht-deutsche Familiensprache eine erhebliche Rolle. In der 1. Klasse der Grundschule in Ludwigshafen, aus deren Unterricht die Beispiele stammen, sprechen die Kinder in ihren Familien neben Deutsch auch Italienisch, Polnisch, Türkisch, Russisch und natürlich Pfälzer Dialekt. Die Kinder sind daher Experten/innenen ihres Dialekts und ihrer nicht-deutschen Familiensprache. Das Handy gibt den Kindern die Chance diese Experten/-innenkompetenz in den Unterricht zu tragen. Um diese Kompetenz in den Erstlese- und Erstschreibunterricht einzubringen, eignen sich Partner/inneninterviews mit dem Handy. In der Lern-Station mit dem Handy in der Übungs- und Vertiefungsphase (siehe Abb. 4) führen die Kinder paarweise ein kurzes Interview, bei dem es um den Laut des neuen Buchstabens $z$ in der deutschen wie auch der Familiensprache geht. Die Kinder bringen als Hausaufgabe ein Wort ihrer Familiensprache mit, in dem der Laut z zu hören ist. Im Interview tauschen sich die Kinder über diese Z-Wörter aus. Ihre Aufgabe: Finde heraus, welches deutsche Wort dem ausländischen entspricht und ob der Laut dann immer noch zu hören ist! Mit der Audiofunktion des Handys nehmen die Kinder das Wort auf und erklären, aus welcher Sprache es kommt und was es bedeutet. Die Kinder bekommen so zum einen ein Gespür für Sprache und zum anderen werden Kinder mit nicht-deutscher Familiensprache und wenig Erfahrungen im Deutschen in dieser Lern-Episode im Deutschunterricht zum Sprachexperten. Kinder nutzen dieses Expertenwissen, um beispielsweise eigene Anlauttabellen in ihrer Muttersprache zu erstellen. 


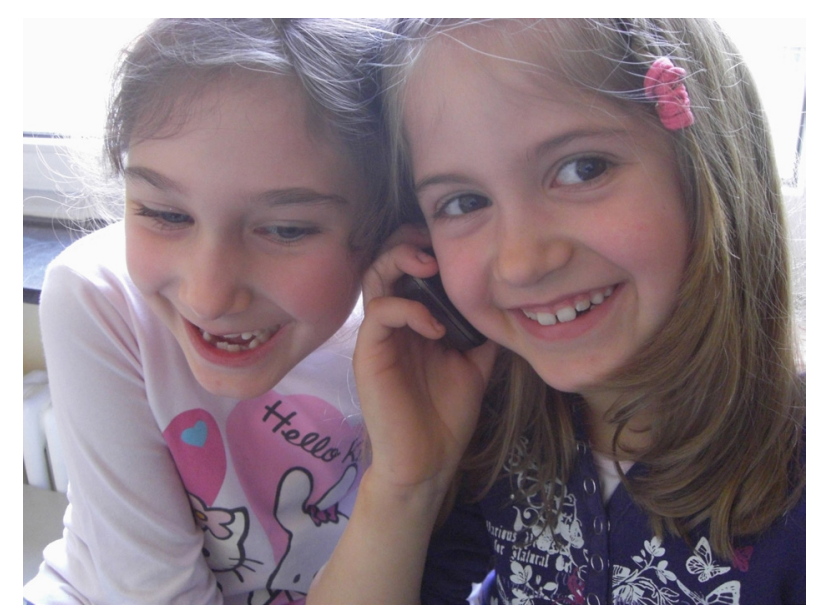

Abbildung 8: Experteninterview anhören Deutsch/GRunDschule

Kinder sind auch Experten/-innen ihres regionalen Dialekts. Im Pfälzischen fällt Kindern die Unterscheidung von sch und ch schwer. Mit dem Handy können sie auf Spurensuche nach dem sch gehen. Die Spurensuche läuft mit Hilfe der Fotofunktion des Handys. Die Kinder fotografieren Dinge und Wörter, die ihnen begegnen und in denen ein sch zu finden ist. Je nach Entwicklungsstand der Kinder und aufgrund der freien Wahlmöglichkeit, was fotografiert wird, entstehen auch Fotos von Dingen, die nur vermeintlich mit einem sch geschrieben werden. Dialekt sprechende Kinder machen aus Kirche oder Licht die Kirsche oder das Lischt. Hier unterstützt die Audiofunktion des Handys das Partner/inneninterview und ist bei der Kontrolle der Aussprache hilfreich. Die Ludwigshafener Kinder sammeln Fotos und Tondokumente von z. B. Milch, Mädchen, Kirche, Licht, Schiff, Schokolade, Schule, Schildkröte, Tisch. In ihrer Sammlung tauchen Wörter auf wie ich, nicht, Vergleich, sechzehn, vielleicht, auch. Es sind Wörter die sich nur mit Hilfe der Audiofunktion sammeln und nicht fotografieren lassen.

\section{Didaktischer Eckpunkt 2.6: Mit dem Handy sensible Entwicklungs- und Lernkontexte schaffen}

Die von Kindern generierten Kontexte der mobilen, individualisierten, konvergenten Medienwelt stehen in der Regel in engem Zusammenhang mit ihren persönlichen Entwicklungsthemen und lassen sich auch nutzbringend in die Lernsituation integrieren. Dabei gilt es genau hinzusehen und zu entscheiden, welche Themen sich zum Nutzen der jeweiligen Kinder aufgreifen lassen. 


\section{Im Mathematikunterricht das Thema Unklarheit aufgreifen}

Bei der Messung von Winkel geht es um Genauigkeit und Eindeutigkeit, was nicht mit der spontanen Herangehensweise von Kindern an Messen und Winkel zusammenpassen muss. Der leitende Gedanke für die sensiblen Entwicklungs- und Lernkontext kommt von Lev Vygotsky (1978 / 1930). Vygotsky ging davon aus, dass Lernen von Sachverhalten nur dann möglich ist, wenn es in die Lebensperspektive der Kinder passt; anders formuliert, wenn die Kinder bereit und in der Lage sind, sich mit Lernthemen zu beschäftigen. Dazu brauchen die Kinder als Schüler/innen unter anderem einen Kontext, der auf ihre Themen und Erlebnisweisen positiv fördernd; mit anderen Worten, der sensibel auf die Kinder und ihre aktuelle Entwicklung eingeht. Das Handy als Teil der Lebenswelt der Kinder ist in der Lage, solch einen sensible Entwicklungs- und Lernkontext mit herzustellen. So hat der Schüler im schon angesprochenen Beispiel seine Winkel-Fotos aus der Hausaufgabe zu einem Video zusammengefügt. Eines dieser Bilder (Abb. 9, linkes Foto) zeigt den 90-Grad-Winkel der Wohnzimmerecke. Auf diesem Foto ist dieser Winkel nur schwer zu erkennen. Mathematisch gesehen taugt dieses Foto wenig als Messergebnis, weil der Winkel nur unscharf und mehrdeutig abgebildet ist. Der Lehrer reagiert positiv unterstützend auf dieses, auf den ersten Blick untaugliche Foto, erkennt doch, dass der Junge mit der Unklarheit von Abbildungen spielt. Der Lehrer sieht die Chance, das Thema der Abbildung und die Mehrdeutigkeit von Abbildungen in den Unterricht hereinzunehmen. Die Frage, weshalb der abgebildete Winkel auf dem Foto nicht mehr mit der Realität übereinstimmt, bekommt in der Episode 6 über Winkel und Perspektiven jetzt auch Raum im Unterricht. Schüler/innen fotografieren die Winkel einer Pyramide aus unterschiedlichen Blickwinkeln (Abb. 9, Foto Mitte). Der Lehrer diskutiert die Ergebnisse auf dem Whiteboard (Abb. 9, rechtes Bild). Mit dem Handy entsteht ein Raum, der von der Zimmerecke zu Hause, über die Erprobung der Perspektiven um Pyramiden-Winkel aufzunehmen bis hin zur Erläuterung durch den Lehrern mit Hilfe des Whiteboards rechts. Dieser Raum funktioniert als Entwicklungsraum, in dem der Junge von seiner unklaren Abbildung den Schritt zu eindeutigen mathematischen Abbildungen mitgehen kann.
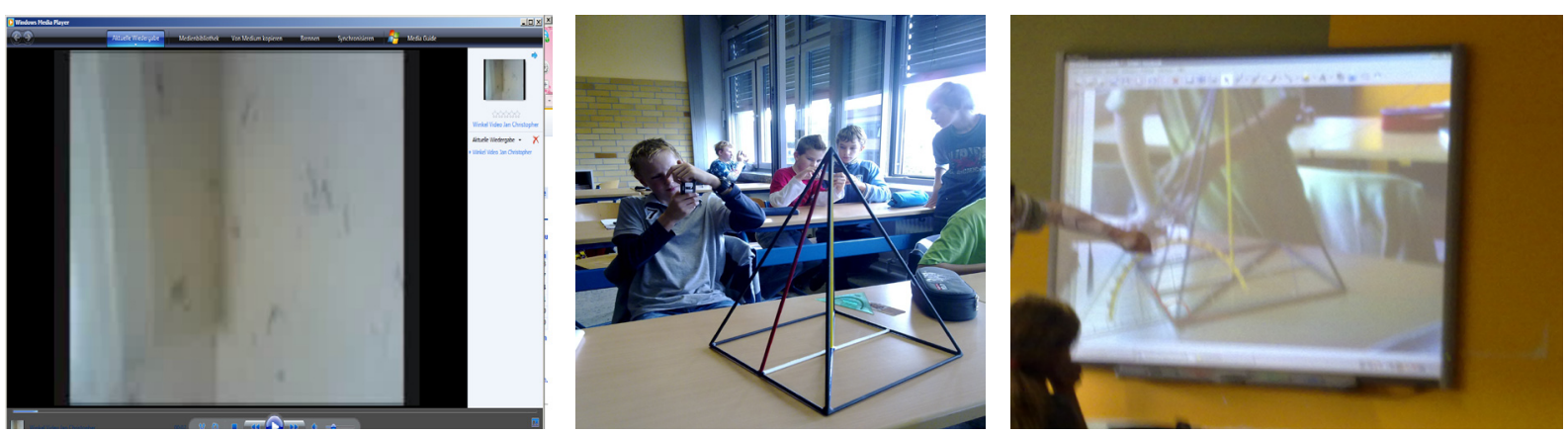

Abbildung 9: Unklarer Winkel als Ergebnis der Hausaufgabe (links); mit Hilfe der Fotografie Perspektiven und Winkel einer Pyramide erkunden (mitte), Fotos von Winkeln am Whiteboard vermessen (rechts) 
In der Episode 6 fotografieren die Schüler/innen Pyramiden-Modelle aus unterschiedlichen Perspektiven (Abb. 9 Mitte). Hier taucht das gleiche Phänomen wie bei der Hausaufgabe auf. Fotos aus unterschiedlichen Perspektiven ergeben unterschiedliche Winkel. Auf dem interaktiven Whiteboard sammeln und erörtern die Schülerinnen und Schüler die Fotos ihrer Gruppenarbeit, die der Lehrer mit ihnen erörtert.

\section{Überblick über die Eckpunkte einer Didaktik des Mobilen Lernens}

Am Schulversuch MyMobile - Handy im Unterricht nahmen sechs Schulen mit unterschiedlich langen Unterrichtseinheiten teil. Dazu gehören die beiden hier skizzierten Unterrichtseinheiten in einer Grundschule und in einem Gymnasium. In diesen beiden Unterrichtseinheiten gab es jeweils sechs Episoden situierten Lernens, in denen das Handy eine tragende Rolle spielte.

Die folgenden Abbildungen 10 und 11 geben einen Überblick über diese HandyEpisoden und die didaktischen Funktionen, die sich mit dem Handy in diesen Episoden realisieren liessen. Die hier kurz skizzierten Eckpunkte einer Didaktik des mobilen Lernens sollen helfen, die didaktischen Funktionen des Handys zu operationalisieren. Auch wenn diese Operationalisierung nur ein erster und heuristischer Versuch einer empirischen Kontrolle ist, so zeigen die beiden folgenden Listen doch die didaktischen Chancen des Handys als Instrument des situierten Lernens.

\begin{tabular}{|c|c|}
\hline Episoden & Eckpunkt \\
\hline 1) Namensbilder & $\begin{array}{l}\text { - Situiertes Lernen (2.2) } \\
\text { - Foto (3.2), Speicher (3.4) }\end{array}$ \\
\hline 2) Auf Spurensuche nach dem "Sch" & $\begin{array}{l}\text { - Situiertes Lernen (2.2), Sensible } \\
\text { Entwicklungs- und Lernkontexte (2.6) } \\
\text { - Foto/Audio (3.2), Speicher ( } 3.4)\end{array}$ \\
\hline 3) Foto-Safari: Elefantenwörter & $\begin{array}{l}\text { - Situiertes Lernen (2.2), Informelles Lernen } \\
\text { integrieren (2.1) in Form von Hausaufgaben } \\
\text { - Foto (3.2), Speicher (3.4) }\end{array}$ \\
\hline 4) Das „Z" - Interview & $\begin{array}{l}\text { - Situiertes Lernen (2.2), Experten des Alltags } \\
(2.5) \\
\text {-Audio (3.2), Speicher (3.4) }\end{array}$ \\
\hline 5) Lischt an oder Licht aus & $\begin{array}{l}\text { - Situiertes Lernen (2.2), Sensible } \\
\text { Entwicklungs-und Lernkontexte (2.6) } \\
\text {-Audio (3.2), Speicher (3.4) }\end{array}$ \\
\hline $\begin{array}{l}\text { 6) Auf Spurensuche nach dem "sp“ - } \\
\text { Bildergeschichten }\end{array}$ & $\begin{array}{l}\text { - Situiertes Lernen (2.2), Sensible } \\
\text { Entwicklungs- und Lernkontexte (2.6) } \\
\text { - Foto/ Audio (3.2), Speicher (3.4) }\end{array}$ \\
\hline
\end{tabular}

Abbildung 10: Überblick über die didaktischen Eckpunkt im Erstlese- und Schreibunterricht der Grundschule

\begin{tabular}{|c|c|}
\hline pisoden & Eckpunkt \\
\hline $\begin{array}{l}\text { Kreis + Kugel: Mathe-Regeln im } \\
\text { Handy speichern }\end{array}$ & -Alltagsexperten (2.5) \\
\hline Vom Kreis zur Kugel & $\begin{array}{l}\text { - informelles Lernen (2.1) } \\
\text { - Kommunikationsbrücke (2.4) } \\
\text { - sensible Entwicklungs-/Lernkontexte (2.6) }\end{array}$ \\
\hline $\begin{array}{l}\text { Foto-Safari:Alttag zwischen Null } \\
\text { und } 360 \text { Grad }\end{array}$ & - Alltagsexperten (2.5) \\
\hline Auf dem Schulhof um den Globus & $\begin{array}{l}\text { - situiertes Lernen (2.2), } \\
\text { - Alltagsexperten (2.5), } \\
\text { - Lern- und Medienkontexte generieren (2.3) }\end{array}$ \\
\hline $\begin{array}{l}\text { Winkel: zwischen Geodreieck und } \\
\text { Video-Quiz/Talk Show }\end{array}$ & $\begin{array}{l}\text { - situiertes Lernen (2.2) } \\
\text {-Alltagsexperten (2.5) }\end{array}$ \\
\hline $\begin{array}{l}\text { Check up: Winkel und } \\
\text { Perspektiven }\end{array}$ & $\begin{array}{l}\text { - Kommunikationsbrücke (2.4), } \\
\text { Alltagsexperte (2.5), sensible } \\
\text { Entwicklungs-/Lernkontexte (2.6), }\end{array}$ \\
\hline
\end{tabular}

Abbildung 11: Überblick über die didaktischen Eckpunkte in der sUnterrichtseinheit über Kreis, Kugel, Winkel im Gymnasium 
Wirft man zusätzlich einen Blick auf die didaktischen Eckpunkte in allen Episoden der bisher ausgewerteten fünf von sechs Projektschulen (Abbildung 12) so zeigt sich, dass sich Eckpunkt 2.2: Situiertes Lernen, sowie Eckpunkt 2.5: Experten des Alltags, am einfachsten in den Unterricht integrieren liessen. Lehrerinnen und Lehrer gelingt es, mit den Handys ihrer Schüler den Unterricht erfolgreich zu öffnen, indem sich Schüler/innen eigenständig mit einem Lernthema befassen und ihr naives, alltägliches Expertenwissen zur individuellen Umsetzung des Lernthemas einsetzen. Auch die sensiblen Entwicklungs- und Lernkontexte (Eckpunkt 2.6) liessen sich in den Unterricht einbinden. Darüber hinaus ist es gelungen, Kommunikationsbrücken und Kommunikationsketten zwischen dem Schüleralltag und dem Unterricht zu schlagen (Eckpunkt 2.4). Für die Schule scheint es dagegen auch mit dem Handy schwierig zu sein, informelles Lernen in die Schule einzubinden (Eckpunkt 2.1: Informelles Lernen in die Schule integrieren).

Informelles Lernen in

Schule integrieren (2.1)

Episoden Situierten

Lernens (2.2)

\section{Lern- und Medienkontexte \\ (2.3)}

\section{Kommunikationsbrücken}

(2.4)

Experten des Alltags (2.5)

\section{Sensible Entwicklungs- und Lernkontexte (2.6)}
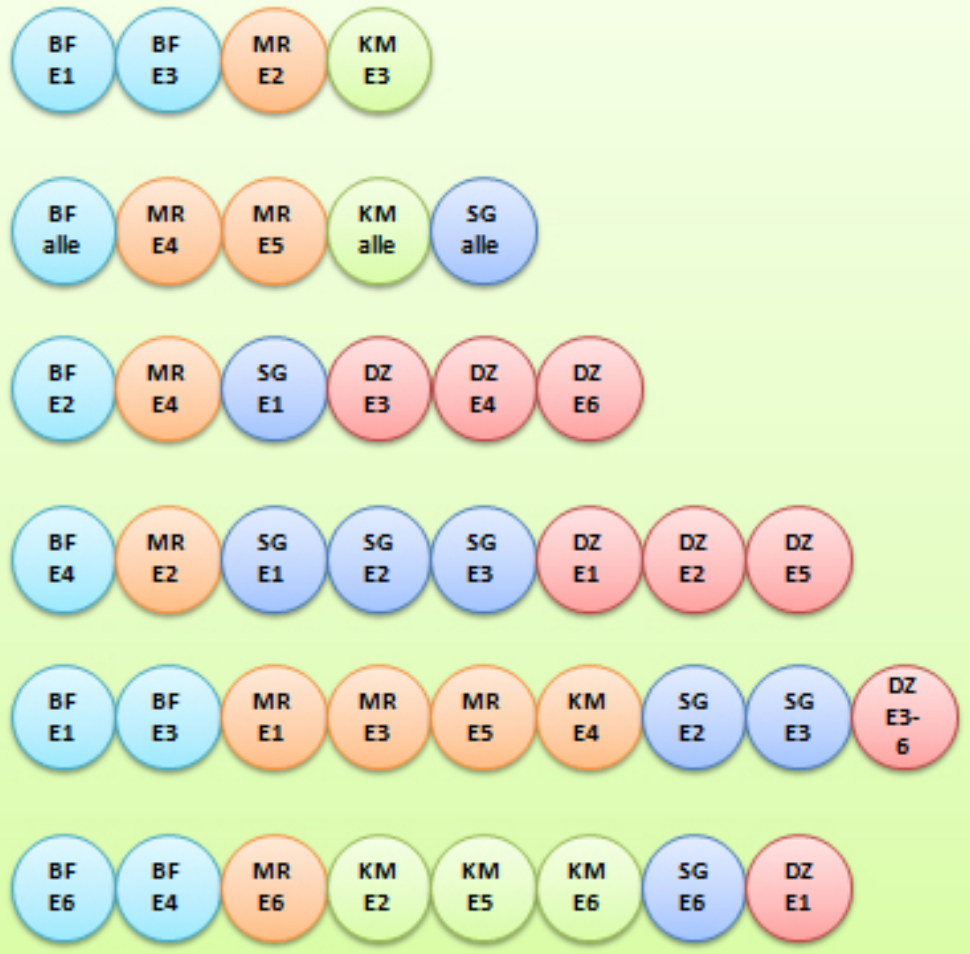

Abbildung 12: Überblick über die didaktischen Eckpunkte aller Unterrichtseinheiten 


\section{Die multimediale Funktionsvielfalt des Handys - sechs Eckpunkte zu den Nutzungsfunktionen des Handys}

In den beiden hier diskutierten Unterrichtseinheiten in der Grundschule und im Gymnasium des Schulversuchs MyMobile - Handy im Unterricht verfügten die Schülerinnen und Schüler über zwei Typen von Handys, zum einen über ihr per-

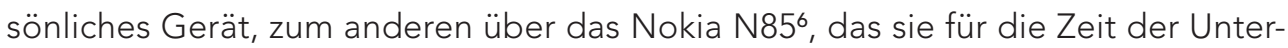
richtseinheit als Projekt-Handys bekamen. Im der sechsten Klasse des Gymnasium hatten die meisten der Schülerinnen und Schüler ein eigenes Handy. In der ersten Grundschulklasse nutzten dagegen alle Kinder in der Schule die Projekt-Handys, bei Hausaufgaben auch die Handys der Eltern.

Ohne die technischen Funktionen eines Handys zu bedenken, lässt sich Unterricht nicht sinnvoll planen. Um die technische Funktionalität zu operationalisieren, gibt es deshalb neben den sechs Eckpunkten zur Didaktik des mobilen Lernens auch sechs Eckpunkt zu den Nutzungsfunktionen des Handys.

Das Handy als mobiler Minicomputer bietet mit seiner Multifunktionsfähigkeit verschiedene Ansatzpunkte für unterschiedliche Lernzwecke. Für die technischen Anwendungen, die heute in einem Handy stecken, hätten Pädagogen/-innen in den 1980er-Jahren eine Vielzahl einzelner Geräte in den Unterricht nutzen müssen. Mit der neuen Generation der Handys stehen breite Nutzungsmöglichkeiten zur Verfügungen. Dabei unterscheiden sich die Handys der Schüler/innen erheblich von einander.

Der Einsatz der Handy-Funktionen in den Episoden der beiden Unterrichtseinheiten von MyMobile - Handy im Unterricht ist vielfältig. Je nach Alter der Schüler/ innen und Lernzweck gibt es unterschiedliche Gewichtungen der Handy-Funktionen. Die Erfahrungen aus den ausgewertete Unterrichtseinheiten von MyMobile - Handy im Unterricht zeigt, dass sich die ständige Verfügbarkeit des Handys (Eckpunkt 3.1) sowie die aktiven Medienfunktionen Foto, Video und Audio (Eckpunkt 3.2) erfolgreich im Unterricht einsetzen lassen. Demgegenüber spielt Eckpunkt 3.5, die Möglichkeiten zur persönlichen Kommunikation mit SMS, eher eine untergeordnete Rolle. Zwar sind gerade diese Funktionen in den ausserschulischen Aktivitäten der Schüler/innen wichtig, ihre Nutzung ist allerdings mit Kosten verbunden, die die Schülerinnen und Schüler im Unterricht vermeiden. Die alltägliche Nutzungsfunktion wie Absprachen zu treffen oder Verabredungen zu vereinbaren, waren für den Unterricht nicht wichtig. Ein Grund dafür ist, dass die Klingeltöne beim Anrufen den Unterricht stören. ${ }^{7}$

6 Diese Entscheidung basiert auf der Empfehlung des «Medienpädagogik Praxisblog» http://www. medienpaedagogik-praxis.de/2009/09/07/das-beste-handy-fur-die-medienpadagogik/

7 Für Hausaufgaben bieten sich sogenannte Cellcast an. Sie wurden jedoch im Rahmen des Schulversuchs MyMobile - Handy im Unterricht nur einmal genutzt. Cellcasts sind digitale Anrufbeantworter im Internet. Die Anmeldung einer solchen Mailbox ist kostenlos. Eine Schulklasse kann mehrere Nummern anmelden und ihre Nachrichten, Fragen oder Interpretation von zuhause aus auf diese Mailbox sprechen. Mehr unter www.cellcast.de. Es entstehen Kosten für ein Gespräch ins Festnetz. 
Die Schülerinnen und Schüler nutzen die Funktionen zur Alltagsorganisation (Eckpunkt 3.3) mit Wecker und Kalender ähnlich häufig wie die hohe Speicherkapazität (Eckpunkt 3.4). Auch das Handy als Schnittstelle zum Internet (Eckpunkt 3.6) war eine im Unterricht genutzte Handy-Funktion. Da der Internetzugang über das Handy zum Teil mit hohen Kosten verbunden ist, reduzierte sich diese Möglichkeit doch deutlich zu Gunsten des Schul-PCs. ${ }^{8}$ Dagegen ist die Konvergenz mit dem interaktiven Whiteboard im Gymnasium wichtig. Als technisch einfache und kostenlose Technik hat sich dabei die Datenübertragung per Bluetooth erwiesen. Besonders die Foto-Ergebnisse aus Kleingruppen lassen sich auf diese Weise zusammenführen. Die Gruppenarbeit mit dem Nokia N85 ermöglicht den direkten Anschluss des Handys mit einem Cinch-Kabel an das Whiteboard. Diese Konvergenz hat sich für das Erarbeiten der Unterrichtsthemen als fruchtbar heraus gestellt.

\section{1 persönliche und ständige Verfügbarkeit \\ 3.2 aktive Medienfunktionen (Video, Foto, Audio)}

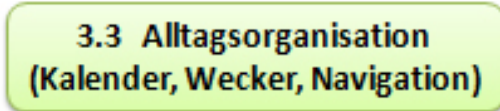

\section{4 hohe Speicherkapazität}

3.5 persönl. Kommunikation (SMS, MMS, Telefon)

\subsection{Konvergenz Internet / Whiteboard}
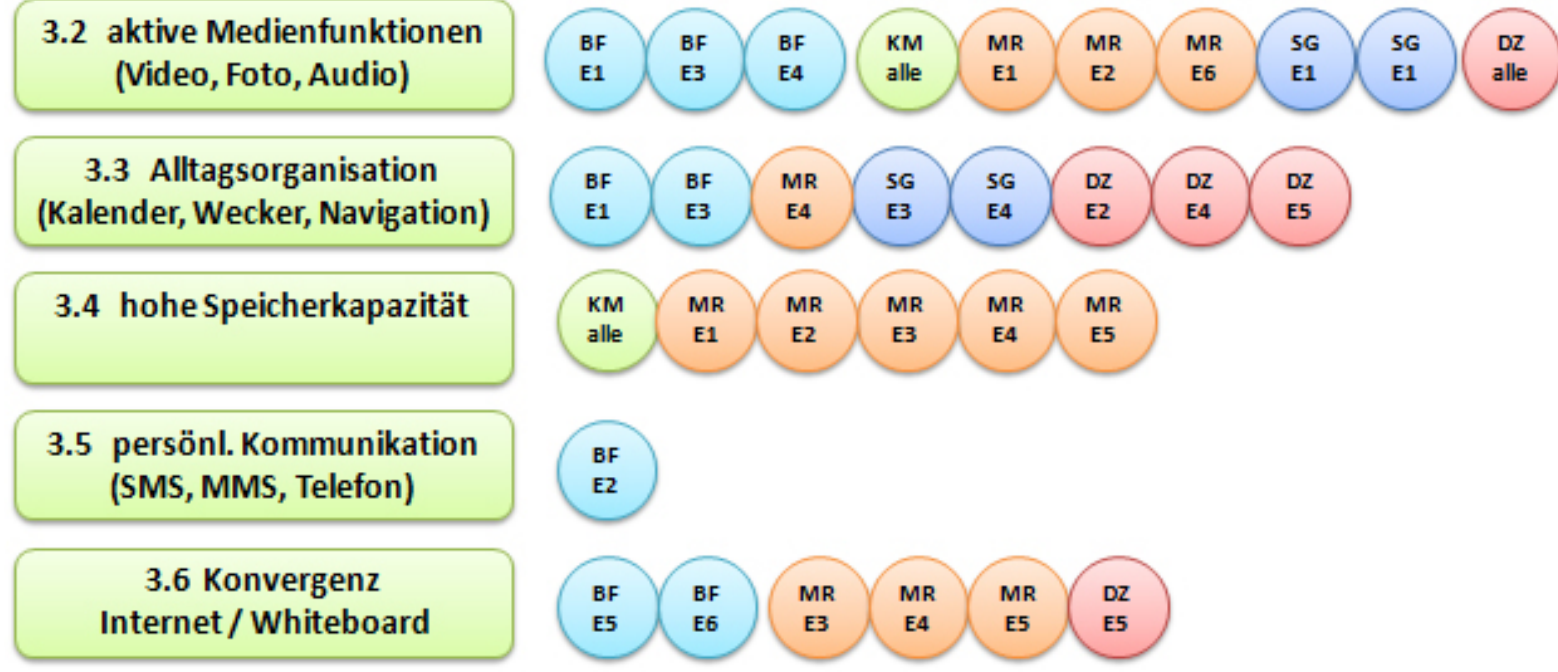

Abbildung 13: Überblick über die Handy-Funktionen aller fünf Ausgewerteten Unterrichtseinheiten Von Mymobile - Handy im Unterricht. Dazu gehören auch die hier diskutierten beiden Unterrichtseinheiten In Der Grundschule (Erstlesen, Erstschreiben) und im Gymnasium (Mathematik)

\section{Eckpunkt 3.1: Persönliche und ständige Verfügbarkeit}

Laut KIM-Studie: Kinder und Medien (Medienpädagogischer Forschungsverbund Südwest 2008), verfügen $19 \%$ der 6- bis 7-Jährigen, $34 \%$ der 8- bis 9-Jährigen, $66 \%$ der 10- bis11-Jährigen und $86 \%$ der 12- bis 13-Jährigen über ein Handy. Die

8 In einer Unterrichtseinheit in einer Hauptschule wurde ein Handy-WLAN-Netz für den Schulversuch eingerichtet. 
gleiche Entwicklung zeigt sich auch in der JIM-Studie von 2009: Jugendliche und Medien (Medienpädagogischer Forschungsverbund Südwest 2009), die die Mediennutzung von 12- bis 19-Jährigen repräsentativ erfasst. $95 \%$ der Jugendlichen haben ein eigenes Handy. Mädchen sind mit $97 \%$ etwas besser ausgestattet als Jungen (93\%). Bereits $88 \%$ der 12- und 13-Jährigen besitzen ein Handy. Bei den über 18-Jährigen herrscht mit 99\% Vollversorgung (14 bis 15 Jahre: 96 \%, 16 bis 17 Jahre: $97 \%)$.

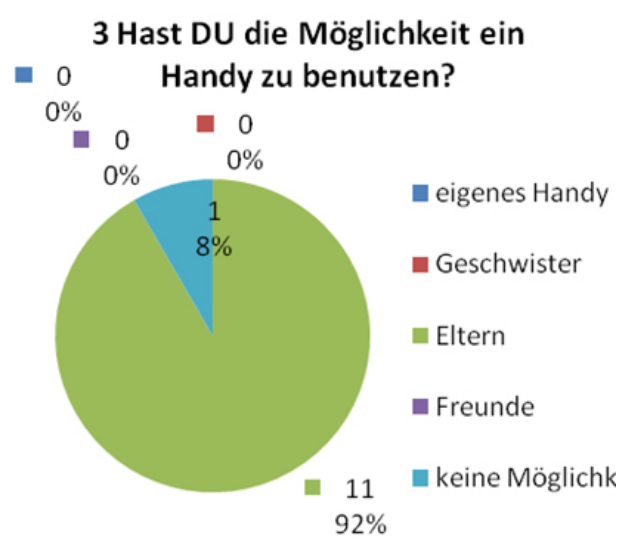

Abbildung 14: Auszug aus der Evaluation Deutsch/ Grundschule

Diese Verteilung des Handys hat sich auch bei der Befragung der Projektklassen von MyMobile - Handy im Unterricht bestätigt. In der sechsten Klasse Mathematik am Gymnasium verfügten die Schüler/innen ( $N=14)$ fast alle (93\%) über ein eigenes Handy (Abbildung 15). Wohingegen in der ersten Klasse der Grundschule lediglich ein Kind ( $N=12)$ angab, ein eigens Handy zu besitzen (Abbildung 14). Deswegen bekamen die Grundschulkinder auch die für den Schulversuch angeschafften sog. Projekt-Handys.

\section{Hast DU die Möglichkeit ein Handy zu benutzen?}

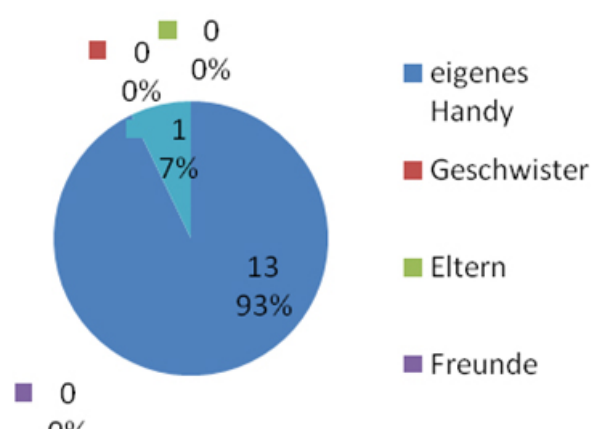

Abbildung 15: Auszug aus der Evaluation Mathematik/ Gymnasium 
Eckpunkt 3.2: Aktive Medienfunktionen für Video, Foto, Audio / Musik

Die aktiven Medienfunktionen des Handys Video, Foto und Audio / Musik, sind bei Kindern und Jugendlichen sehr beliebt und werden häufig genutzt. Die Evaluation von MyMobile - Handy im Unterricht zeigt, dass Kinder der sechsten Klasse Mathematik diese Funktionen annährend gleich oft nutzen (Abbildung 16).

Obwohl die Grundschüler/innen in der Regel über kein eigenes Handy verfügen, sind sie mit den aktiven Medienfunktionen der Projekt-Handys schnell vertraut, nicht zuletzt weil sie vor allem die Fotokamera in den Handys der Eltern schon häufiger genutzt haben (Abbildung 17). Mit diesen Erfahrungen nutzen die Schülerinnen und Schüler die aktiven Medienfunktionen im Unterricht auch häufig (vgl. Abbildung 13). Die meisten Handys verfügen mittlerweile über eine einfach zu bedienende Foto- und Videokamera, die gute Qualität liefert. Das Handy ermöglicht aber auch Varianten wie das Einsprechen von Sprachmemos oder die Übertragung von Bildern via Bluetooth. Diese technischen Varianten erweitern in höheren Klassenstufen das Handlungsfeld für Schülerinnen und Schüler signifikant. In der Grundschule waren vor allem Foto- und Audiofunktion von zentraler Bedeutung, weil Foto und Audio für jüngere Kinder intuitiv bedienbar und wenig fehleranfällig sind. Warum keine Digitalkamera statt Handy verwenden? Der Vorteil des Handys liegt in der Breite der aktiven Medienfunktionen in einem Gerät. Schüler/innen können deshalb viel flexibler einzelne Funktionen nutzen.

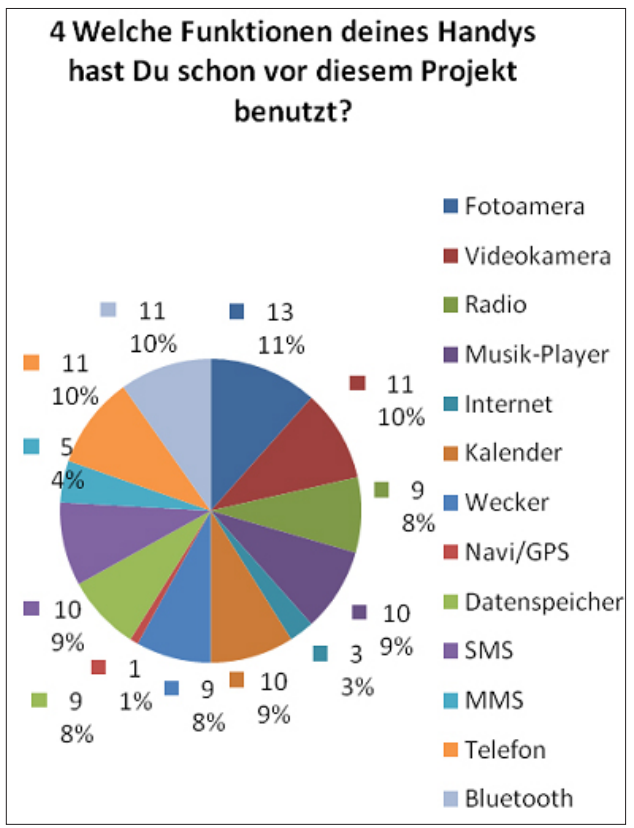

Abbildung 16: Auszug aus der Evaluation Mathematik im Gymnasium

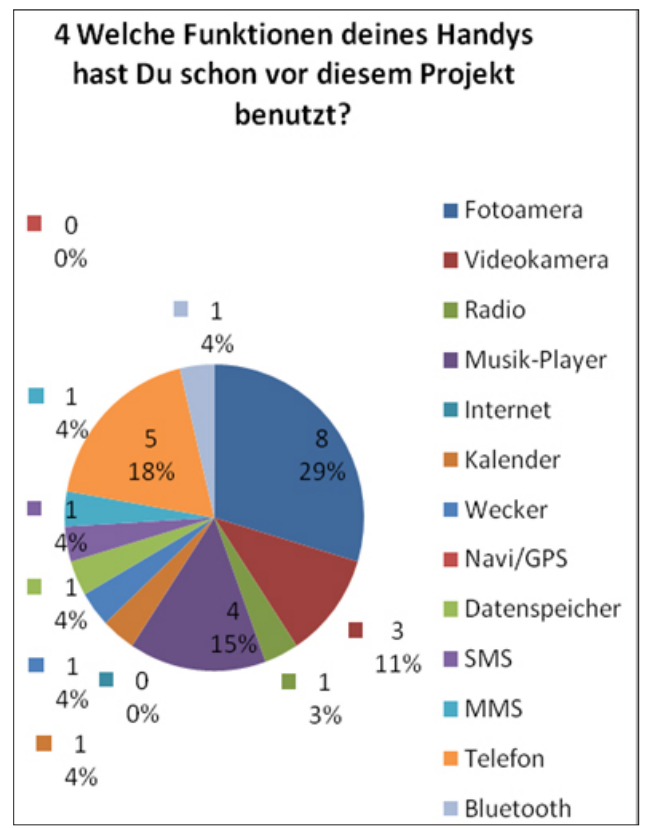

Abbildung 17: Auszug aus der Evaluation des Erstlesens und-schreibens in der Grundschule 


\section{Fotografiere Deine Welt! - Die Fotofunktion in der Grundschule}

Die Fotokamera des Handys dient als Lupe oder Fernglas, um die Umwelt zu erkunden und zu entdecken. Die Kinder erhalten einen Arbeitsauftrag ("Fotografiere Dinge, in denen ein Sch vorkommt») und ziehen anschliessend selbstständig mit dem Handy los. Die so von den Kindern produzierten Fotos bieten Anlässe für Gespräche. Sie zeigen auch den unterschiedlichen Entwicklungsstand der Kinder auf. Die Lehrerin integriert die entstandenen Fotos in Form von Arbeitsblättern in den Unterricht. Die Kinder sind motiviert, weil sie ihre eigenen Fotos wieder entdecken und zum gemeinsamen Lernen einsetzen. Das führt zu einem höheren Lernerfolg. Die Kinder arbeiten nicht nur am vorgegebenen Stoff im Schulbuch oder auf dem Arbeitsblatt, sondern an ihren eigenen Fotos zu Anfangs-, Mittel- und Endlauten. Die bekannten Gegenstände aus dem unmittelbaren Umfeld der Kinder werden damit zu Lernhilfen. Kinder verstehen sehr schnell, was sich auch ausserhalb der Schule entdeckend lässt. Sie vertiefen durch diese Aktivitäten spielerisch auch ausserhalb der Schule die Lernthemen (siehe Abb. 18).
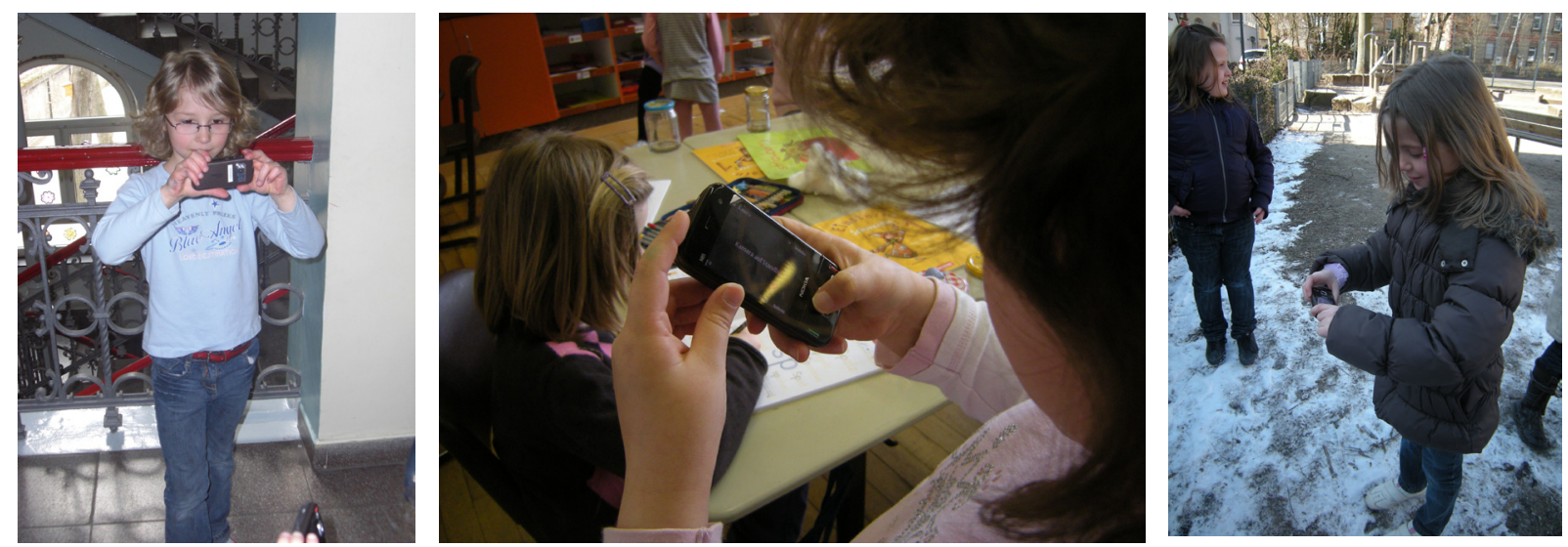

Abbildung 18: Fotografieren mit dem Handy in der Grundschule

Wie hört sich das an? - Die Audiofunktion in der Grundschule

Grundschulkinder nutzen die Audiofunktion des Handys vor allem passiv als Musikplayer (vgl. Abbildung 17). Eines der Ziele der Medienkompetenzförderung besteht darin, Medien auch aktiv zu verwenden. Deshalb geht es beim Erstlesen und Erstschreiben auch darum, den Erstklässlern/-innen diese Funktion nahe zu bringen. Die eigenen Lese- und Hörversuche bei Elefantenwörtern oder von Wörter mit sch, die sie fotografiert hatten, nehmen die Kindern schnell und begeistert an. Auf der Suche nach Gegenständen oder Begriffen mit bestimmten Buchstaben oder Lauten, fallen manchen Kindern Begriffe ein, die sie nicht als Foto festhalten können, da sie nicht im Schulhaus zur Verfügung stehen, zum Beispiel Apfelsaft- 
schorle, Schädel, Fisch. Mithilfe der Audiofunktion sprechen die Kinder diese Begriffe auf das Handy und tragen sie mit dem Handy in den Unterricht.

In der Grundschule gibt es einige Kinder, die mit dem Schrifterwerb Schwierigkeiten haben und streckenweise nicht schreiben wollen. Diese Kinder bekommen mit dem Handy einen alternativen Zugang zu den relevanten Wörtern und Lauten. Sie analysieren die gehörten Wörter, indem sie sie aussprechen und dabei die Stellung verschiedener Buchstaben hörend kontrollieren. Mit dieser Hör-Analyse lässt sich auch die Familiensprache und der regionale Dialekt in den Unterricht hereinnehmen, indem andere Schülerinnen und Schüler oder die Lehrerin einen Ausspracheunterschied thematisieren und dann die richtige Schreibweise an der Tafel festhalten. Beim Einsatz der Audiofunktion fiel auf, dass sonst eher stille Kinder sich aktiv beteiligen und begeistert Wörter in das Handys sprachen. In der Episode 4, beim Z-Interview, bekommt die Audiofunktion eine Schlüsselfunktion (siehe Abb. 17).
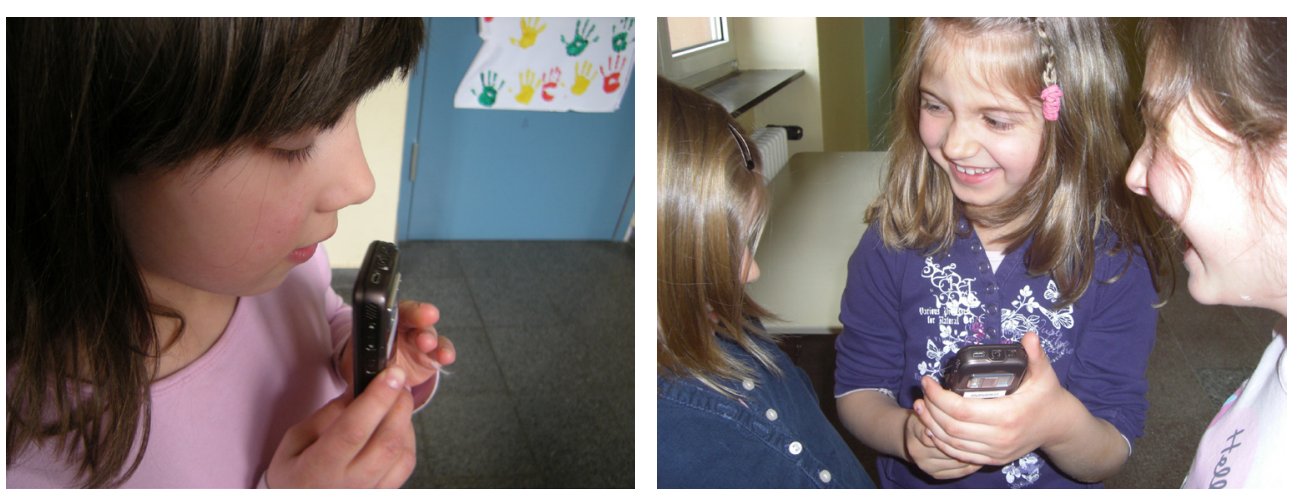

Abbildung 19: Sprachaufnahmen in der Grundschule

\section{Eckpunkt 3.3: Anwendungen für die Alltagsorganisation: Kalender, Wecker und Navigation}

In den Bereich der Alltagsorganisation des Handys fallen Anwendungen wie Kalender, Wecker, auch der Rechner und die Navigation. Im Alltag nutzen die Schüler/innen diese von Handys unterschiedlich angebotenen Funktionen. Von den 12-jährigen Schüler/innen im Gymnasium geben 3\% Internetzugang an. 8\% sagen, sie nutzen den Wecker und $1 \%$ gibt an, Navi / GPS haben (Abb. 16). Die Grundschulkinder sagen, dass sie zu 4\% den Wecker, 0\% das Navi / GPS und 4\% den Kalender verwenden (Abb. 17).

In vielen der Episoden setzten die Schüler/innen die Stoppuhr ihres Handys ein, um ihre Zeit für die Einzel- oder Gruppenarbeit festzulegen. 


\section{Auf dem Schulhof um die Welt - GPS im Mathematik-Unterricht}

Der Mathematik-Unterricht der 6. Klasse beschäftigt sich u. a. mit den Lernthemen Vom Kreis zur Kugel sowie Winkel zeichnen und messen. In diesen Lernzusammenhang gehören auch Kugelkoordinaten (Lergenmüller, Schmitt S. 49, Aufgabe 19). Mit Hilfe von GPS können die Schüler/innen den praktischen Nutzen von Kugelkoordinaten im Alltag ausprobieren und den Transfer zur Mathematik leisten. Die Unterrichtsstunde leitet der Lehrer ein. Im Anschluss folgt eine handygestützte Gruppenarbeit auf dem Schulhof, bei der die Schüler/innen selbst Koordinaten über GPS suchen. In dieser Phase verbindet sich das Handy mit Satelliten und empfängt die Position der Schüler/innen auf dem Schulhof. Zurück im Klassenzimmer geben die Gruppen ihre Daten über das interaktive Whiteboard in die Internetanwendung Google Earth ein und prüfen ihre Daten darauf hin, ob sie korrekt sind (siehe Abb. 7).

\section{Eckpunkt 3.4: Hohe Speicherkapazität für vielfältige Darstellungsformen}

Handys verfügen neben einem internen Datenspeicher auch über auswechselbare Speicherkarten. Diese Speicherkarten, sogenannte Micro-SD- oder SD-Karten sind in verschiedenen Grössen verfügbar und standardmässig im Lieferumfang der Handys enthalten. Diese Speicherkarten fungieren ähnlich wie USB-Sticks und ermöglichen auch das Speichern von grösseren Datenmengen. Der Austausch von Dateien wie Foto-, Video- und Audioformaten ist mit dieser erweiterbaren Speicherkapazität komfortabel und technisch meist einfach zu handhaben. Aber auch ohne diese Erweiterungen bietet die Mehrzahl der Handys die für Bild- und Videodateien notwendige grosse Speicherkapazität. In der ersten Klasse der Grundschule gibt es deswegen auch keine technischen Grenze, als Kinder sich ihren Wortschatz in ihrer Lebenswelt suchten und dabei in Episode 2 beliebig viele geschrieben Wörter mit dem sch fotografierten, in Episode 3, der Foto-Safari, Elefantenwörter (zusammengesetzte Nomen-Wörter) entdeckten und fotografierten. In Episode 2 bringt ein Mädchen gleich 15 Fotos von zuhause mit. In Episode 3 präsentiert ein Junge stolz seine mitgebrachten Fotos mit den extrem langen Substantiven Abfallwirtschaftsgesellschaft und Müllheizkraftwerk (siehe Abb. 3). Auf diese Elefantenwörter war er beim Wochenendspaziergang mit dem Vater gestossen. Das väterliche Handy erlaubt es dem Sohn, neu entdeckte komplizierte Wörter mit in die Schule zu bringen. Aus seinen Handyfotos wählt er zwei aus und präsentiert sie in der Klasse. In Episoden 4 (Z-Interview, das Z in den Familiensprachen und im Dialekt) sammeln die Kinder mit der Audiofunktion gesprochene Wörter. In Episode 5 (Lischt an oder Licht aus?) gibt es für die Schüler/innen-Interviews mit der Audio-Funktion des Handys ebenfalls keine technischen Begrenzungen. 
Eckpunkt 3.5: Zugang zu einer vielfältigen persönlichen Kommunikation (SMS, MMS, Telefon)

Bei den sechs Unterrichtseinheiten des Schulversuches MyMobile - Handy im Unterricht sollte den Schülerinnen und Schülern keine Kosten für Telefonieren, SMS oder MMS entstehen, weshalb sie, falls sie keine Flat-Rate hatten, die Projekt-Handys nutzten. Daten übermittelten sie per Datenkabel oder Bluetooth. Telefonate waren während des Unterrichts wegen der damit einhergehenden Störung nicht erwünscht. Schülerinnen und Schüler verfassten mit der Notizfunktion Nachrichten und verschickten sie in dieser Form kostenlos über Bluetooth. Die Notizfunktion vieler Handy sieht aus wie die bekannten gelben Klebe-Notizzettel Post-it. So notierten sich Schülerinnen und Schüler z. B. ihre Mathematik-Hausaufgaben mit der Notizfunktion auf dem Handy und leiteten sie auch an Mitschüler/innen weiter. Im Deutschunterricht der ersten Klasse fanden SMS, MMS und Notizfunktion keine Verwendung.

Eckpunkt 3.6: Zugang zum Internet mit dessen spezifischer Kommunikation und dessen Informations- und Medienarchiven sowie zu anderen Schnittstellen wie dem interaktiven Whiteboard

In Episode 5 des Mathematikunterrichts im Gymnasium liessen sich die Kugelkoordinaten der Erde nur mit Hilfe der Konvergenz von Handy und Internet auf dem Schulhof erkunden. Im Grundschulunterricht der 1. Klasse wurde der Zugang zum Internet nicht eingesetzt. Dafür waren zwei Gründe massgeblich. Zum einen hatten die Grundschulinder keine internetfähigen Handys, zum anderen fehlte den Erstklässlern/-innen die notwendige technische und kritische Internetkompetenz. In einer Hauptschule in einer der anderen hier nicht vorgestellten Unterrichtseinheiten von MyMobile - Handy im Unterricht gab es einen speziellen WLAN-Zugang für die Handys.

Im Mathematikunterricht des Gymnasiums reduzierte sich der Trend zum medienkonvergenten Handy auf das interaktive Whiteboard im Klassenzimmer. Der Lehrer nutzte das interaktive Whiteboard in jeder Unterrichtstunde. Für die Episoden situierten Lernens war der Einsatz des interaktiven Whiteboard wesentlich für:

- Sichten und Vermessen von Winkeln (Episode 3),

- Nutzen von Google Earth für das Erarbeiten der Kugel.-Koordinaten (Episode 4),

- Vermessen von Winkel in unterschiedlichen Perspektiven (Episode 6),

- Sammeln von mathematischen Regeln (Episoden 1 und 2) und gemeinsames Anschauen von Videos (Episode 5).

Die Schüler/innen verwenden im Unterricht oder bei der Hausaufgabe die Fotofunktion des Handys. Das dabei entstandene Bildmaterial präsentieren sie in der der Klasse auf dem interaktiven Whiteboard. 


\section{Operationalisierung des mobilen Lernens an Regelschulen: eine zusammenfassende Bewertung}

Bei der Operationalisierung des mobilen Lernens geht es sowohl um eine didaktische Systematik also auch um deren praktische Anwendung für Lehrerinnen und Lehrer in der Alltagsroutine der Regelschule. Mittel der Operationalisierung waren die beiden Listen von Eckpunkten der Planung und Analyse des mobilen Lernens, und zwar die Eckpunkte Didaktik und die Eckpunkte Handy-Anwendungen. Obwohl die beiden hier vorgestellten Unterrichtseinheiten sehr unterschiedlich waren, sowohl in Bezug auf Altersgruppe und Schultyp (erste Klasse Grundschule; zweite Klasse im Gymnasium) als auch für die didaktische Praxis in beiden Schulen (Stationen-Lernen; vor allem angeleiteter Frontalunterricht), funktionierten die Eckpunkte Didaktik und Handy-Anwendungen erfolgreich als Hilfsmittel für die Verständigung mit Lehrerin und Lehrer bei der Planung des Handy-Einsatzes. Am Ende der Unterrichtseinheit gaben Lehrerin und Lehrer sowie die Schülerinnen / Schüler ihre Einschätzung des Handy-Einsatzes ab.

Wo liegen die Grenzen dieses Vorgehens? Das didaktische Design, Episoden situierten Lernens mit dem Handy in den lehrergeleiteten Unterricht einzufügen, akzeptiert die Art, wie Schule Lernen und Lehren organisiert. Die didaktische Grundüberlegung richtet sich auf die Assimilation des vertrauten Mediums Handy oder das Handy als Alltagsinstruments in den bekannten und von einer Lehrerin oder einem Lehrer geleiteten Unterricht. Die Episoden situierten Lernens liefern die Möglichkeit, den Prozess der didaktischen Assimilation zu bewerkstelligen. Bei diesem didaktischen Design mit dem eindeutigen Vorrang für den bekannten Schulunterricht, sind dann alltagstypische Formen des Lernens, insbesondere beiläufiges und informelles Lernen, eher institutionell benachteiligt. Deswegen fiel es bei den Unterrichtseinheiten auch schwer, Formen des informellen Lernens zu entdecken. Informelles, beiläufiges, unauffälliges Lernen ist jedoch mit dem Alltagsinstrument Handy verbunden, so die These, weil auch der Alltag von Lernen durchdrungen ist. Um bei dem didaktischen Assimilations-Design schuluntypische Lernformen zu entdecken, war eine systematische Auswertung der Unterrichtseinheiten notwendig, bei der das Team der Medienpädagogen/-innen und Medienpädagogen/-innen mit Hilfe der Eckpunkte auch die nicht offensichtlichen Ereignisse des Unterrichts diskutierten. Die Beschränkung der Diskussion auf das Medienpädagogik-Team ist dem Praxisdruck und den begrenzten Personalressourcen von Schule geschuldet. In den Unterrichtseinheiten gab es Team-Unterricht, sodass die Klassenlehrerinnen und Klassenlehrer keinen wesentlichen zusätzlichen Vor- oder Nachbereitungsaufwand treiben mussten. Der Team-Unterricht war jedoch nicht formalisiert worden, sondern folgte Ad-hoc-Absprachen, was mit einer Ausnahme, die beteiligten Lehrer/innen und Medienpädagogen/-innen bzw. den männlichen Kollegen auch als erfolgreich bewerteten. 
Mit Hilfe der im ersten Teil skizzierten didaktischen Parameter lassen sich die Unterrichtseinheiten auch knapp allgemein bewerten. Charakteristisch für beide Unterrichtseinheiten war, das Handy als Alltagsinstrument der Schülerinnen und Schüler in den Regelunterricht einzubinden. Regelunterricht ist eine Lehr- und Lernform, bei der ein Lehrer bzw. Lehrerin die Kinder anleitet (Parameter 1), in dem sich die Schülerinnen und Schüler den Lerngegenstand vor allem reproduzierend und mimetisch aneignen (Parameter 2). Massgeblich ist dabei der Lehrplan (Parameter 3). Medien tauchen vor allem als eigenständige, diskrete Einheit wie das Schulbuch auf (Parameter 4). Der Regelunterricht ordnet sich als vor allem den linksseitigen Polen der Parameter zu. Das situierte Lernen lieferte in beiden Unterrichtseinheiten der Regelschule die Chance, den jeweiligen Gegenpol des entsprechenden Parameters in die Schule hereinzunehmen. Das Model für den Gymnasialunterricht lieferte das Bild der Lern-Strasse, auf der der Lehrer das Lernen anleitet. Dabei liegen, wie für die Regelschule typisch, die Schwerpunkte der vier Parameter bei den Polen Schulpraxis, Schulcurriculum, Reproduktion des Lerngegenstandes und der isoliert linearen Mediennutzung. Die in diese Lern-Strassen eingestreuten LernPlätze oder Lernepisoden des situieren Lernens helfen dann jedoch die Gegenpole der vier Parameter zu realisieren.

Im Grundschulunterricht war die Idee des situierten Lernens in der Form des Stationen-Lernens als massgeblicher Teil der Lehr- und Lernpraxis vorhanden. Das Handy als eine Lern-Station neben mehreren anderen öffnete den Unterricht jedoch für die Pole Alltag (Parameter 1), subjektive Rekonstruktion (Parameter 2), subjektive Kompetenz von Schülern als naive Experten (Parameter 3). In der Unterrichtseinheit liessen sich die Grundschülerinnen auf ihren Sprachalltag ein und gingen im Schulumfeld und zu Hause mit den Eltern auf die Suche nach Wörtern und Lauten. Die Rekonstruktion ihres Sprechens im Kontext der Familiensprache bekommt mit dem Handy den Charakter des Normalen.

Im Mathematikunterricht des Gymnasiums sind Alltag, subjektive Rekonstruktion, subjektive Kompetenz von Schülern/-innen als naive Experten/-innen eher ebenso randständig wie das situierte Lernen, das diese Pole zu realisieren hilft. In einem Unterricht mit einer deutlichen traditionellen Ausprägung in Bezug auf die Parameter bekam das Handy die wichtige didaktische Funktion, Lern-Episoden zu ermöglichen. Dazu bot sich vor allem das Handy als Hausaufgabe an. Trotzdem braucht es zusätzliche Beratung des Lehrers und Unterstützung der Schülerinnen und Schüler, um die subjektive Rekonstruktion z. B. von Winkel im eigenen Wohnumfeld mit Pop-Musik auch mit den die Forderungen des Lehrplans zu verbinden. Die Handy-Funktion, mit der man sich ins digitale geographische Kartennetzwerk einklinken kann, war dagegen einfach zu realisieren. Hier ist der Bezug zum Lerngegenstand ähnlich offensichtlich wie bei der Verzerrung von Winkel beim Fotografieren. Mit einer deutlichen Nähe zum Lerngegenstand öffnen sich dann auch Verbindungen zwischen der Fotofunktion des Handys und den typischen mathe- 
matischen Repräsentationsmitteln wie dem Modell einer Pyramide aus stabilem Material. Das Handy zusammen mit der digitalen Tafel, dem Whiteboard, bietet sich im Moment als vorrangige Form der Medienkonvergenz in der Schule an. Diese Konvergenz mit dem Whiteboard trägt deutlich dazu bei, mit dem Handy Formen des situierten Lernens zu realisieren. Das Handy im Kontext des situieren Lernens erhöht die Möglichkeit, Alltag (Parameter 1), subjektive Rekonstruktion (Parameter 2) und die subjektiven Kompetenzen der Schülerinnen und Schüler (Parameter 3 ) in den Unterricht zu integrieren.

\section{Literaturnachweis}

Bachmair, Ben (2009). Medienwissen für Pädagogen. Medienbildung in riskanten Erlebniswelten. VS Verlag für Sozialwissenschaften, Wiesbaden

Bachmair, Ben, Cook, John, Pachler, Norbert (2009). Mobile phones as cultural resources of learning, an educational analysis of structures, mobile expertise and cultural practices. In: MedienPädagogik Feb. 2009. www.medienpaed.com

Bertelsmann Stiftung, Initiativkreis Bildung unter der Schirmherrschaft des Bundespräsidenten (1999). Zukunft gewinnen - Bildung erneuern: Memorandum. Verlag Bertelsmann Stiftung, Gütersloh ISBN 3-89204-456-2

BILD-ARCHIV, Rundschau über Lichtbild / Film / Druckbild im Dienste de Jugenderziehung u. Volksbildung. Verlag Schule u. Kunst, München. Herausgegeben von L.M.K. Capeller, Pasing. Heft 1, 1920

Böhme, Janette (2006). Schule am Ende der Buchkultur. Medientheoretische Begründungen schulischer Bildungsarchitektur. Klinkhardt Verlag, Bad Heilbrunn

Brown, Ann L. (1992). Design Experiments: Theoretical and Methodological Challenges in Creating Complex Interventions in Classroom Settings. The Journal of the Learning Sciences, 2(2) 141-18. Lawrence Erlbaum Ass

Brown, John Seely, Collins, Allan, Duguid, Paul: Situated Cognition and the culture of learning. In Educational Researcher 18 (1), 1989, S. 32-42

Dourish, P. (2004). 'What we talk about when we talk about context.' In Personal and Ubiquitous Computing 8(1), pp. 19-30. Verfügbar unter http://www.ics.uci. edu/ jpd/publications/2004/PUC2004-context.pdf

Habermas, Jürgen (1990). Strukturwandel der Öffentlichkeit. Neuwied 1962, Vorwort zur Neuauflage, Suhrkamp, Frankfurt

Hanks, William F. (1990). Foreword. In: Lave, Jean, Wenger, Etienne: Situated learning: Legitimate peripheral participation. Cambridge University Press., Cambridge, S. 13-24

Hasebrink, Uwe, Mikos, Lothar, Prommer, Elisabeth (2004) (Hrsg.). Mediennutzung in konvergierenden Medienumgebungen. Verlag Reinhard Fischer, München

Jörissen, Benjamin (2007). Informelle Lernkulturen in Online-Communities. Mediale Rahmungen und rituelle Gestaltungsweisen (2007). In: Wulf, Christoph, Althans, Birgit u. a. (2007) (Hrsg.). Lernkulturen im Umbruch. Rituelle Praktiken 
in Schule, Medien, Familie und Jugend. VS Verlag für Sozialwissenschaften, Wiesbaden, S. 184-219

Kleinsteuber, Hans J., Thomass, Barbara (2004). Globalisierung des Medienmarktes. Entwicklung. Strukturen, Risiken. In: Kübler, Hans-Dieter, Elling, Elmar (Hrsg.): Wissensgesellschaft. Neue Medien und ihre Konsequenzen. Bundeszentrale für politische Bildung, Bonn 2004, S. 31-42

Kerres, Michael (2005). Gestaltungsorientierte Mediendidaktik und ihr Verhältnis zur Allgemeinen Didaktik. In: Stadtfeld, Peter, Dieckmann, Bernhard (Hrsg.): Allgemeine Didaktik im Wandel. Julius Klinkhardt, Bad Heilbrunn, S. 214-234

Kress, G. (2010). Learning and Environments of Learning in Conditions of Provisionality. In: Bachmair, B. (ed.) (2010). Medienbildung in neuen Kulturräumen. Die deutschsprachige und britische Diskussion. VS Verlag für Sozialwissenschaften, Wiesbaden, S. 171-182

Kron, Friedrich, W., Sofos, Alivisos (2003). Mediendidaktik. Neue Medien in Lehrund Lernprozessen. Ernst Reinhardt Verlag, München Basel

Laurillard, D. (2007) 'Pedagogical forms of mobile learning: framing research questions.' In: Pachler, N. (ed) Mobile learning: towards a research agenda. WLE Centre, Institute of Education, London, pp. 153-176. Verfügbar unter: http://www.wlecentre.ac.uk/cms/files/occasionalpapers/mobilelearning_pachler_2007.pdf

Lave, Jean, Wenger, Etienne (1991). Situated learning: Legitimate peripheral participation. Cambridge University Press, Cambridge

Lergenmüller, Arno, Schmitt, Günter (2006). Mathematik, Neue Wege, Arbeitsbuch für Gymnasien, 6. Schuljahr, Kreis und Winkel, Winkel schätzen und messen. Westermann, Braunschweig

Medienpädagogischer Forschungsverbund Südwest (2008). KIM-Studie: Kinder und Medien. http://www.mpfs.de/index.php?id=133

Medienpädagogischer Forschungsverbund Südwest (2009). JIM-Studie: Jugend und Medien. http://www.mpfs.de/index.php?id=161

Mikos, Lothar (2004). Die Aufwertung des Alltags. Events, Spassgesellschaft und die ,Inszenierung des Banalen'. In: Kübler, Hans-Dieter, Elling, Elmar (Hrsg.): Wissensgesellschaft. Neue Medien und ihre Konsequenzen. Bundeszentrale für politische Bildung. Bonn 2004, S. 54-59

Ministerium für Bildung, Frauen und Jugend Rheinland-Pfalz (2005): Rahmenplan Grundschule, Teilrahmenplan Deutsch, Mainz

Pachler, N., Bachmair, B., Cook, J. (2010). Mobile Learning: Structures, Agency, Practices. New York: Springer

Pachler, Norbert, Cook, John, Bachmair, Ben (2010). Appropriation of mobile cultural resources for learning. In: International Journal for Mobile and Blended Learning, Vol. 2, No. 1, 2010, January-March 2010. PP. 1 - 21. IGI Global. Hershey PA, USA. ISSN 1941-8647. eISSN 1941-8655 
Patten, Bryan, Arnedillo-Sánchez, Inmaculada, Tangney, Brendan (2006). Designing collaborative, constructionist and contextual applications for handheld devices: Virtual Learning? In: Computers \& Education, 46 (3), p. 294-308

Sacher, Werner (2005). Didaktik als Theorie des arrangierten Lernens. In: Stadtfeld, Peter, Dieckmann, Bernhard (Hrsg.): Allgemeine Didaktik im Wandel. Julius Klinkhardt, Bad Heilbrunn, S. 173-213

Tervooren, Anja (2007).Tanz, Prüfung und Wettkampf. Lernkulturen jugendlicher Mädchen zwischen Ent- und Reritualisierung. In: Wulf, Christoph, Althans, Birgit u. a. (2007) (Hrsg.): Lernkulturen im Umbruch. Rituelle Praktiken in Schule, Medien, Familie und Jugend. VS Verlag für Sozialwissenschaften, Wiesbaden, S. 253-287

Tulodziecki, Gerhard, Herzig, Bardo unter Mitarbeit von Silke Grafe und Maria Herrlich (2004): Mediendidaktik. Band 2. Handbuch Medienpädagogik. KlettCotta, Stuttgart

Vygotsky, L. (1978 / 1930) Mind in society. The development of higher psychological processes. Edited by M. Cole et al., Harvard University Press, Cambridge, MA.

Vygotsky, L. (1986/1934) Thought and language. Edited by A. Kozulin, MIT Press, Cambridge, MA. 